\title{
Composition-Aware Spectroscopic Tomography
}

\author{
Luke Pfister $^{1}$, Rohit Bhargava ${ }^{1,2,3,4,5}$, Yoram Bresler $^{1}$, and P. Scott Carney ${ }^{6}$ \\ ${ }^{1}$ Department of Electrical and Computer Engineering, University of Illinois at \\ Urbana-Champaign, Urbana, Illinois 61801, USA \\ ${ }^{2}$ Department of Bioengineering, University of Illinois at Urbana-Champaign, Urbana, Illinois \\ 61801, USA \\ ${ }^{3}$ Department of Mechanical Science and Engineering, University of Illinois at \\ Urbana-Champaign, Urbana, Illinois 61801, USA \\ ${ }^{4}$ Department of Chemical and Biomolecular Engineering, University of Illinois at \\ Urbana-Champaign, Urbana, Illinois 61801, USA \\ ${ }^{5}$ Department of Chemistry, University of Illinois at Urbana-Champaign, Urbana, Illinois 61801, \\ USA \\ ${ }^{6}$ The Institute of Optics, University of Rochester, Rochester, New York 14627, USA
}

\begin{abstract}
Chemical imaging provides information about the distribution of chemicals within a target. When combined with structural information about the target, in situ chemical imaging opens the door to applications ranging from tissue classification to industrial process monitoring. The combination of infrared spectroscopy and optical microscopy is a powerful tool for chemical imaging of thin targets. Unfortunately, extending this technique to targets with appreciable depth is prohibitively slow.

We combine confocal microscopy and infrared spectroscopy to provide chemical imaging in three spatial dimensions. Interferometric measurements are acquired at a small number of focal depths, and images are formed by solving a regularized inverse scattering problem. A low-dimensional signal model is key to this approach: we assume the target comprises a finite number of distinct chemical species. We establish conditions on the constituent spectra and the number of measurements needed for unique recovery of the target. Simulations illustrate imaging of cellular phantoms and sub-wavelength targets from noisy measurements.
\end{abstract}

\section{Introduction}

Chemically specific imaging provides quantitative information about the distribution of chemicals within a target. This may be accomplished through the use of exogenous chemicals or molecular staining to improve contrast when the target is imaged with visible light. In many applications, these dyes cannot be introduced in situ and the agents are often damaging to the target.

Vibrational spectroscopy with mid-infrared light presents a solution [1]. Absorption of mid-infrared light depends on chemical composition. The underlying chemistry of a target can be determined, non-invasively, by illuminating the object with mid-infrared light and recording an absorption spectrum.

In principle, mid-infrared spectroscopy can provide chemically specific, spatially resolved imaging in three spatial dimensions using a confocal scanning strategy: the target would be scanned point-by-point in three spatial dimensions, and an absorption spectrum would be measured at each point [2, 3]. For a target with two spatial dimensions, this is feasible- a typical data set of 1024 spectral samples over a $1024 \times 1024$ pixel grid requires on the order an of hour of acquisition time and generates roughly 4 GB of data $4 .-7]$. Scanning along a third spatial dimension (depth) makes imaging even a single target impractical: the resulting dataset would require over 4 terabytes of storage and roughly a month of acquisition time.

The key challenge in jointly measuring structural and chemical information is dimensionality: with no constraints, the target can vary in three spatial and one spectral dimension. Existing imaging modalities explicitly or implicitly rely on simple signal models to reduce the dimensionality of the target and allow for practical imaging. 
Optical Coherence Tomography (OCT) and Interferometric Synthetic Aperture Microscopy (ISAM) are scatteringbased imaging modalities that reconstruct the 3D spatial distribution of a target by ignoring spectral variation [8-11] , although limited spectral information can be recovered at the expense of spatial resolution by way of timefrequency analysis $[12-14]$.

Fourier Transform Infrared (FTIR) spectroscopy, a workhorse of academic and industrial labs worldwide, neglects all spatial variation within the target—-thus reducing the target to a single dimension. An extension, FTIR microspectroscopy, provides spatially and spectrally resolved measurements but requires the target to be very thin with only transverse heterogeneities. Unmodeled spatial variations in the target cause scattering and diffraction, ultimately distorting the measured spectra 2.3$]$.

We propose an approach that bridges these two extremes and allows for practical, chemically specific imaging. We call this spectroscopic tomography. Rather than finely scanning the focus through the axial dimension of the target, we acquire data at a small number of en-face focal planes. The target is recovered by solving the linearized scattering problem. A low-dimensional model is used to regularize the inverse problem: we model the target as the linear combination of a finite number of distinct chemical species. We call this the $N$-species approximation. We develop a set of algebraic conditions for unique recovery and examine the conditioning of the inverse problem. Reconstructions from synthetic phantom data illustrate the promise of the model.

The $N$-species model was investigated for a one-dimensional target in [15]. However, [15] involved several involve several unrealistic assumptions, leading to results of unrealistically high quality. We extend this work in several directions: we (i) use a non-asymptotic forward model; (ii) demonstrate material-resolved reconstruction of samples with two spatial dimensions (one transverse and depth, easily extended to three spatial dimensions) from synthetic scattering data that are not generated according to the first Born approximation; and (iii) refine the conditions for recovery of a sample consisting of $N$-species from interferometric scattering experiments.

The paper is organized as follows. In Section 2 we describe the forward model. Section 3 describes the $N$ species model in greater detail. We discuss the sampling and discretization procedure in Section 4 We investigate the inverse problem in Section 5 and demonstrate the method by performing numerical reconstructions from simulated measurements in Section 6 ,

\subsection{Notation}

We write the set of integers $\{0,1, \ldots, N-1\}$ as $[N]$ and the two-fold Cartesian product $[N] \times[N]$ as $[N]^{2}$. We write the imaginary unit as i. Finite-dimensional vectors are denoted by lower-case bold letters, e.g. $\mathbf{x} \in \mathbb{C}^{N}$. Finitedimensional matrices and multi-dimensional arrays are written using upper-case bold letters. We adopt Matlabstyle indexing notation: given a matrix $\mathbf{A} \in \mathbb{C}^{N \times M}$, its $i$-th row is $\mathbf{A}[i,:]$, the $j$-th column is $\mathbf{A}[:, j]$, and the $(i, j)$-th element is $\mathbf{A}[i, j]$. We denote the vector $\operatorname{vec}(\mathbf{A}) \in \mathbb{C}^{N M}$ is formed by stacking the columns of $\mathbf{A}$ into a single vector (i.e., row-major ordering). The range, null space, and rank of a matrix $\mathbf{A}$ are written $\operatorname{range}\{\mathbf{A}\}$, null $\{\mathbf{A}\}$, and $\operatorname{rank}(\mathbf{A})$. Given $\mathbf{x} \in \mathbb{C}^{N}$, the diagonal matrix $\operatorname{diag}\{\mathbf{x}\} \in \mathbb{C}^{N \times N}$ has the entries of $\mathbf{x}$ along its main diagonal.

The transpose (resp. Hermitian transpose) of a matrix is written $\mathbf{A}^{\top}\left(\right.$ resp. $\left.\mathbf{A}^{\mathrm{H}}\right)$. The $\ell_{p}$ norm of $\mathbf{x} \in \mathbb{C}^{N}$ is $\|\mathbf{x}\|_{p}=\left(\sum_{j=1}^{N}|\mathbf{x}[j]|^{p}\right)^{1 / p}$. For vectors in $\mathbb{R}^{2}$ or $\mathbb{R}^{3}$ we use the shorthand $|r|=\|\mathbf{r}\|_{2}$. The $N \times N$ identity matrix is $\mathbf{I}_{N}$, and the vector $[1,1, \ldots 1]^{\top} \in \mathbb{R}^{N}$ is written $\mathbb{1}_{N}$. The tensor (or Kronecker) product between matrices $\mathbf{A}$ and $\mathbf{B}$ is $\mathbf{A} \otimes \mathbf{B}$.

\section{Preliminaries}

We characterize the sample under investigation by its complex refractive index, $n(\mathbf{r}, k)=n_{b}+\delta n(\mathbf{r}, k)$ where $n_{b}$ is the refractive index of the background medium and $\delta n$ is the perturbation due to the sample; for simplicity, we take $n_{b}=1$. Here, $\mathbf{r}=(x, y, z)=(\boldsymbol{\rho}, z)$, where $\boldsymbol{\rho}$ are the transverse dimensions and $z$ indicates the axial dimension. We assume that $\delta n$ is (spatially) supported in the bounded region $\Gamma \subset \mathbb{R}^{3}$. The free-space wavenumber $k$ is related to temporal frequency $\omega$ by $k=\omega / c$, where $c$ is the speed of light in free space. The real part of the complex refractive index is the ratio between $c$ and the phase velocity in the medium, while the imaginary part indicates attenuation due to propagation through the target.

Under the first Born approximation, the obtained measurements are linear in the complex susceptibility $\eta \triangleq$ $n^{2}-1$; we will work with the susceptibility rather than the refractive index. Note that $\eta$ is also supported on $\Gamma$.

In the context of spectroscopy, the "spectrum" of a sample usually refers either to its complex refractive index or only the imaginary part of the refractive index. Consider a homogeneous medium with refractive index $n(k)=$ 


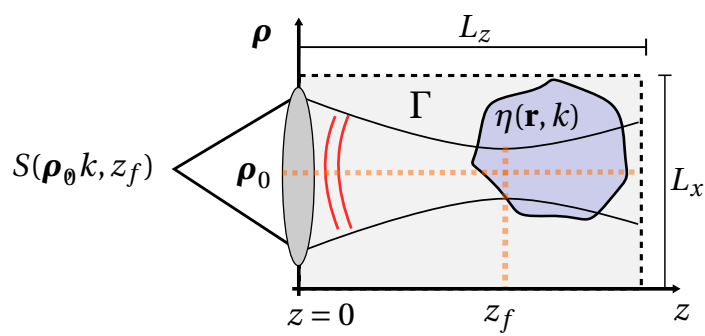

Figure 1: Geometry and notation for scattering problem. The illuminating aperture is located at $\left(\boldsymbol{\rho}_{0}, 0\right)$. The field emerges from the aperture and is focused to the plane $z=z_{f}$. The incident beam interacts with the sample, $\eta$, and the backscattered light (red) is collected through the aperture to produce the measurement $S\left(\boldsymbol{\rho}_{0}, k, z_{f}\right)$.

$n_{r}(k)+\mathrm{i} \kappa(k)$. The real part, $n_{r}(k)$, has mean value greater than one and the imaginary part, $\kappa(k)$, is non-negative. Relating $\eta(k)$ to $n(k)$, we have

$$
\eta(k)=n(k)^{2}-1=n_{r}(k)^{2}-\kappa(k)^{2}-1+2 \mathrm{i} n_{r}(k) \kappa(k) .
$$

Unlike the refractive index, the mean value of the real part of $\eta(k)$ may be less than one and can be negative. The imaginary part of $\eta(k)$ remains non-negative.

\subsection{Interferometric Synthetic Aperture Microscopy}

In this section, we review the forward model relating the target, $\eta$, to the observed data. For a complete derivation, see \begin{tabular}{l|l|l|l|l|l|l|}
\hline & 11 & 16 & 17 & .
\end{tabular}

The imaging geometry is depicted in Fig. 1. An aperture located at $\left(\boldsymbol{\rho}_{0}, 0\right)$ emits a broadband Gaussian beam focused to a point $\mathbf{r}_{0}=\left(\boldsymbol{\rho}_{0}, z_{f}\right)$ within the sample. The illuminating field interacts with the sample, and a portion of the light is scattered backwards and is collected through the aperture. The aperture is raster scanned along the transverse coordinates $\boldsymbol{\rho}_{0}$. At each point the scattered field is measured interferometrically, from which we use standard techniques to recover the complex (phase-resolved) measurements. In the remainder of this paper, we ignore the interferometric aspects of data acquisition and work directly with the phase-resolved measurements.

Under the first Born approximation, the measured data $S\left(\boldsymbol{\rho}_{0}, k, z_{f}\right)$ are a linear function of $\eta$; we have

$$
S\left(\boldsymbol{\rho}_{0}, k, z_{f}\right)=\iint A\left(\boldsymbol{\rho}_{0}-\boldsymbol{\rho}, z-z_{f}, k\right) \eta(\boldsymbol{\rho}, z, k) \mathrm{d} z \mathrm{~d}^{2} \rho,
$$

or, after taking a Fourier transform with respect to the scanning dimension $\boldsymbol{\rho}_{0}$,

$$
\hat{S}\left(\boldsymbol{\kappa}, k, z_{f}\right)=\frac{1}{2 \pi} \int S\left(\boldsymbol{\rho}_{0}, k, z_{f}\right) e^{-\mathrm{i} \boldsymbol{\kappa} \cdot \boldsymbol{\rho}_{0}} \mathrm{~d}^{2} \rho=\int \hat{A}\left(\boldsymbol{\kappa}, z-z_{f}, k\right) \hat{\eta}(\boldsymbol{\kappa}, z, k) \mathrm{d} z .
$$

We call the function $\hat{A}$ the ISAM kernel. This function is itself defined by an integral; explicitly,

$$
\hat{A}(\boldsymbol{\kappa}, z, k) \triangleq \frac{|\zeta(k)|^{2}}{k^{2} \mathrm{NA}^{2}} \int_{\Omega(\boldsymbol{\kappa}, k)} \frac{\exp \left\{-\frac{1}{(k \mathrm{NA})^{2}}\left(\left|\boldsymbol{\kappa}^{\prime}\right|^{2}+\left|\boldsymbol{\kappa}-\boldsymbol{\kappa}^{\prime}\right|^{2}\right)+\mathrm{i} z\left(k_{z}\left(\boldsymbol{\kappa}^{\prime}, k\right)+k_{z}\left(\boldsymbol{\kappa}-\boldsymbol{\kappa}^{\prime}, k\right)\right)\right\}}{k_{z}\left(\boldsymbol{\kappa}^{\prime}, k\right)} \mathrm{d}^{2} \boldsymbol{\kappa}^{\prime},
$$

where $k_{z}(\boldsymbol{\kappa}, k) \triangleq \sqrt{k^{2}-|\boldsymbol{\kappa}|^{2}}$ and the set $\Omega(\boldsymbol{\kappa}, k) \triangleq\left\{\boldsymbol{\kappa}^{\prime} \in \mathbb{R}^{2}:\left|\boldsymbol{\kappa}-\boldsymbol{\kappa}^{\prime}\right| \leq k,\left|\boldsymbol{\kappa}^{\prime}\right| \leq k\right\} \subset \mathbb{R}^{3}$ restricts the integral to propagating modes. The scalar NA $>0$ is the numerical aperture of the illumination lens and $|\zeta(k)|^{2}$ is the power spectrum of the illumination source. We assume that $\zeta(k)$ is supported on the interval $\left[k_{\min }, k_{\max }\right]$

\subsection{Image Reconstruction using ISAM}

Next, we discuss recovering the object $\eta$ from measurements of the form 2]. First, note that $\zeta(k)$ in 3 ensures that $\hat{A}(\boldsymbol{\kappa}, z, k)$ vanishes for any $k \notin\left[k_{\min }, k_{\max }\right]$. Further, $\Omega(\boldsymbol{\kappa}, k)$ is empty for $|\boldsymbol{\kappa}|>2 k$ and so $\hat{A}(\boldsymbol{\kappa}, z, k)$ vanishes for all $|\boldsymbol{\kappa}|>2 k_{\max }$. Thus the measurements are related to the bandlimited transverse Fourier transform of the object. 


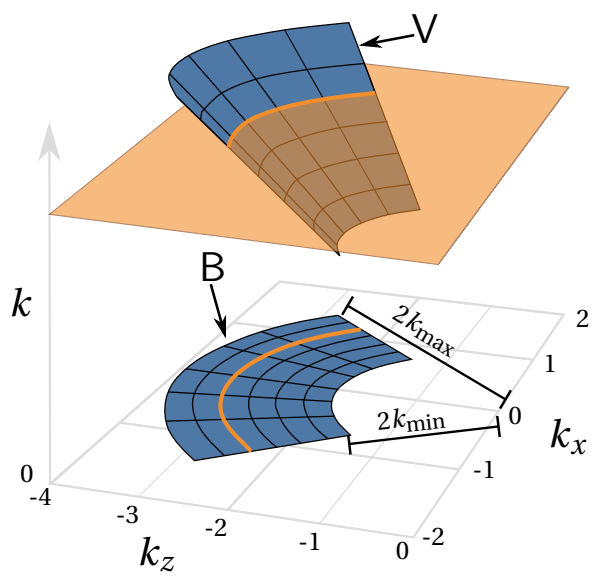

Figure 2: Observable Fourier components for a target with two spatial and one spectral dimensions. The intersection of $\mathrm{V}$ with a plane of constant $k$ becomes an arc of constant radius when projected onto the $\left(k_{x}, k_{z}\right)$ plane.

Previous derivations of ISAM continue by invoking a pair of approximations to the integral 3. One approximation holds when $k\left|z-z_{f}\right|$ is small and the other holds when the same quantity is large. Both approximations are of the form

$$
\hat{A}\left(\boldsymbol{\kappa}, z-z_{f}, k\right) \approx \chi(\boldsymbol{\kappa}, 2 k)|\zeta(k)|^{2} \vartheta(\boldsymbol{\kappa}, k) v\left(z-z_{f}\right) e^{\mathrm{i} k_{z}(\boldsymbol{\kappa}, 2 k)\left(z-z_{f}\right)}
$$

where

$$
\chi(\boldsymbol{\kappa}, k) \triangleq \begin{cases}1, & |\boldsymbol{\kappa}| \leq k \\ 0, & \text { otherwise }\end{cases}
$$

the function $\vartheta(\boldsymbol{\kappa}, k)$ captures the transverse bandpass nature of the imaging system due to the aperture, and $v(z)$ is a depth-dependent weighting function. The precise form of these functions depends on if $k\left|z-z_{f}\right|$ is large or small; in either case, $\vartheta(\kappa, k) \propto e^{-\frac{|\boldsymbol{\kappa}|^{2}}{(k \mathrm{NA})^{2}}}$ and $v(z)$ falls off as $z^{-1}$ 11.

Inserting (4) into the measurement model 2], we have

$$
\hat{S}\left(\boldsymbol{\kappa}, k, z_{f}\right) \approx \chi(\boldsymbol{\kappa}, 2 k)|\zeta(k)|^{2} \vartheta(\boldsymbol{\kappa}, k) e^{\mathrm{i} k_{z}(\boldsymbol{\kappa}, 2 k) z_{f}} \int v\left(z-z_{f}\right) \hat{\eta}(\boldsymbol{\kappa}, z, k) e^{-\mathrm{i} k_{z}(\boldsymbol{\kappa}, 2 k) z} \mathrm{~d} z .
$$

Consider a single, fixed, focal plane; this is the usual setting for ISAM imaging. Define the weighted susceptibility

$$
\hat{\xi}_{z_{f}}(\boldsymbol{\kappa}, z, k) \triangleq v\left(z-z_{f}\right) \hat{\eta}(\boldsymbol{\kappa}, z, k) .
$$

The integral in 5 is the Fourier transform of $\hat{\xi}_{z_{f}}$ with respect to $z$ evaluated at the frequency $-k_{z}(\boldsymbol{\kappa}, 2 k)$; thus

$$
\hat{S}\left(\boldsymbol{\kappa}, k, z_{f}\right) \approx \chi(\boldsymbol{\kappa}, 2 k)|\zeta(k)|^{2} \vartheta(\boldsymbol{\kappa}, k) e^{\mathrm{i} k_{z}(\boldsymbol{\kappa}, 2 k) z_{f}} \hat{\hat{\xi}}_{z_{f}}\left(\boldsymbol{\kappa},-k_{z}(\boldsymbol{\kappa}, 2 k), k\right),
$$

where the double hat indicates a 3D Fourier transform with respect to $\mathbf{r}=(x, y, z)$. This is a generalized projectionslice theorem: the ISAM data are approximately the bandlimited (spatial) Fourier transform of the weighted susceptibility evaluated on a three dimensional surface that is parameterized by $\boldsymbol{\kappa}$ and $k$. By varying $\boldsymbol{\kappa}$ and $k$, we are able to observe a curved 3D "slice" of the four-dimensional function $\hat{\hat{\xi}}_{z_{f}}\left(\boldsymbol{\kappa}, k_{z}, k\right)$ constrained to the surface

$$
\mathrm{V} \triangleq\left\{\left(k_{x}, k_{y}, k_{z}, k\right): \sqrt{k_{x}^{2}+k_{y}^{2}+k_{z}^{2}}=2 k, k_{z}<0, k_{x}^{2}+k_{y}^{2} \leq 4(k \mathrm{NA})^{2}, k_{\min } \leq k \leq k_{\max }\right\} .
$$

The sampling surface for a target with two spatial dimensions, i.e. $\mathbf{r}=(x, z)$, is illustrated in Fig. 2 that we can only observe $k_{z}<0$ is due to the backscattering geometry. As defined, $\mathrm{V}$ contains only the Fourier components above the $e^{-2}$ cutoff frequency of $\vartheta(\boldsymbol{\kappa}, k)$. This is arbitrary as $\vartheta(\boldsymbol{\kappa}, k)$ decays smoothly.

We cannot recover an arbitrary object given ISAM data at a single focal plane. If the focus $z_{f}$ were scanned in addition to $\boldsymbol{\rho}_{0}$, we could further simplify by taking a Fourier transform along $z_{f}$. Then, the measurements would 
be of the form $\hat{\hat{S}}\left(\boldsymbol{\kappa}, k_{z}, k\right)=\hat{\hat{A}}\left(\boldsymbol{\kappa}, k_{z}, k\right) \hat{\hat{\eta}}\left(\boldsymbol{\kappa}, k_{z}, k\right)$, where the double hat indicates the 3D Fourier transform with respect to $\mathbf{r}$. Now, $\eta$ could be recovered using a standard deconvolution procedure. Unfortunately, this is infeasible for reasons described in Section 1

The situation is simplified if $\eta$ is not a function of $k$; such an object is said to be non-dispersive. This is one of the key assumptions on which ISAM, OCT, diffraction tomography, and reflection tomography are built $\left[\begin{array}{l|l|l|l|}\hline & 18 & \text {. In }\end{array}\right.$ this case, the measurements are related to a 3D slice of the 3D target $\eta(x, y, z)$. The observable Fourier components are

$$
\mathrm{B} \triangleq\left\{\left(k_{x}, k_{y}, k_{z}\right): \sqrt{k_{x}^{2}+k_{y}^{2}+k_{z}^{2}}=2 k, k_{z}<0, k_{x}^{2}+k_{y}^{2} \leq 4(k \mathrm{NA})^{2}, k_{\min } \leq k \leq k_{\max }\right\}
$$

The region B is called the optical passband of the ISAM imaging system. Strictly speaking, we observe the Fourier components of the weighted susceptibility on $\mathrm{B}$, but this distinction is usually ignored. Only a non-dispersive (weighted) object whose spatial Fourier transform is supported on B can be perfectly imaged by the ISAM system with a single focal plane. Otherwise, ISAM is able to recover, at best, a spatial bandpass version of the original target. In the visualization of Fig.2. B is the "shadow" cast by $\mathrm{V}$ onto the plane $k=0$.

We do not directly use the approximate kernel (4) in this paper. However, we use the insight provided by this approximation as a guide; in particular, the Fourier transform interpretation and the optical passband B inform the sampling procedure and help establish fundamental limits of the imaging system.

\section{The $N$-species Model}

\subsection{The Model}

Existing imaging modalities use simplified signal models to reduce the dimensionality of the sample and allow for practical imaging. ISAM, optical coherence tomography, diffraction tomography, and reflection tomography require the target be either non-dispersive or have known spatially invariant dispersion characteristics. In this case, the susceptibility is of the form $\eta(\mathbf{r}, k)=p(\mathbf{r}) h(k)$, where $p(\mathbf{r})$ captures the spatial density of the target and $h(k)$ characterizes the wavelength-dependent dispersion characteristics. If $h(k)$ is known, only $p(\mathbf{r})$ must be determined - thus reducing the problem to recovery of a three-dimensional object. Conversely, Fourier Transform Infrared spectroscopy of a bulk medium assumes that the sample is spatially homogeneous, so that $\eta(\mathbf{r}, k)=h(k)$. An extension, FTIR microscopy, models the sample as a thin absorbing screen; thus $\eta(\mathbf{r}, k)=\eta(\boldsymbol{\rho}, k)$, a threedimensional object.

These examples severely restrict the class of samples that can be imaged. We propose a model that is more expressive than these examples while still allowing practical imaging.

Definition 1 (The $N$-species model [15]). An object, described by a susceptibility $\eta(\mathbf{r}, k)$, is said to satisfy the $N$ species model if

$$
\eta(\mathbf{r}, k)=\sum_{s=1}^{N_{s}} p_{s}(\mathbf{r}) h_{s}(k)
$$

The function $p_{s}(\mathbf{r})$ captures the spatial variation of the $s$-th species and is called the spatial density. If species $s$ is not present at location $\mathbf{r}$, then $p_{s}(\mathbf{r})=0$. The complex function $h_{s}$ models the wavelength-dependent properties of the $s$-th species and is called the spectral profile.

The $N$-species model, introduced in [15], is a rank $N_{s}$ approximation to a general susceptibility. A similar decomposition has been applied to magnetic resonance spectroscopic imaging, where it is called the Partially Separable (PS) function model [20-23], and to material decomposition in X-ray tomography [24 25].

\subsection{Spectroscopic Tomography with the $N$-Species Model}

Inserting the $N$-species model [6] into the linearized forward model 2], we have

$$
\hat{S}\left(\boldsymbol{\kappa}, k, z_{f}\right)=\sum_{s=1}^{N_{s}} h_{s}(k) \int_{-\infty}^{\infty} \hat{A}\left(\boldsymbol{\kappa}, z-z_{f}, k\right) \hat{p}_{s}(\boldsymbol{\kappa}, z) \mathrm{d} z
$$


At a given focal plane, the measurements are the sum of $N_{s}$ independent ISAM experiments, each on a nondispersive object $\hat{p}_{s}(\boldsymbol{\kappa}, z)$ and each weighted by the spectral profile $h_{s}(k)$. In what follows, we study inverse problem associated with spectroscopic optical tomography: we wish to recover an object that satisfies the $N$-species model from measurements of the form 7 .

In the single species case, the inverse problem can be solved from data acquired at single focal plane-this is the usual ISAM problem. On the other hand, an arbitrary sample can be recovered by finely scanning in all three spatial dimensions (i.e., along $\boldsymbol{\rho}_{0}$ and $z_{f}$ ) and acquiring a spectrum at each point, but this is infeasible as described in Section 1

The $\mathrm{N}$-species model is a middle ground between a single species object and an arbitrary one. We show that the number of measurements required to solve the inverse problem also lies in a middle ground between these two extremes: in particular, an object satisfying the $N$-species model can be recovered using $N_{f} \approx N_{s}$ focal planes.

We divide the inverse problem into three distinct cases.

(P1) Known Spectra. Assume the spectral profiles $\left\{h_{s}\right\}_{s=1}^{N_{s}}$ are fixed and known. The task reduces to a linear inverse problem—recovery of the $\left\{\hat{p}_{s}\right\}_{s=1}^{N_{s}}$ from measurements of the form 7 .

(P2) Spectra from a Dictionary. Assume the target comprises at most $N_{s}$ chemical species, but the spectral profiles are drawn from a (known) dictionary of some $M_{s}>N_{s}$ possible spectra. The inverse problem can be phrased as either a linear inverse problem over the entire dictionary, or as a nonlinear problem where the solution is constrained to lie in a union of subspaces.

(P3) Fully Blind. Both the $\left\{h_{s}\right\}_{s=1}^{N_{s}}$ and $\left\{\hat{p}_{s}\right\}_{s=1}^{N_{s}}$ are unknown and must be recovered from measurements of the form 7). This is a bilinear inverse problem in $h_{s}$ and $p_{s}$.

In this paper, we limit our attention to cases (P1) and (P2) Our analysis is based on a discretized form of 7 . wherein all quantities are replaced by finite-dimensional versions, resulting in a so-called "discrete-to-discrete" inverse problem [26 27]. Next, we describe the sampling and discretization procedure.

\section{Sampling and Discretization of the Forward Model}

\subsection{Sampling}

The instrument acquires samples of the spatial-domain measurement equation (1). We assume the object is (spatially) supported in a region $\Gamma \subset \mathbb{R}^{3}$; here, we take $\Gamma=\left[0, L_{x}\right] \times\left[0, L_{y}\right] \times\left[0, L_{z}\right]$. We write the number of samples as $N_{i}$ and the discretization or sampling interval as $\Delta_{i}$ for $i \in\{x, y, z, k\}$. We obtain measurements at the transverse aperture locations $\boldsymbol{\rho}_{0}=\left(n_{x} \Delta_{x}, n_{y} \Delta_{y}\right)$ for integers $n_{x}, n_{y}$. The parameters are chosen to cover $\Gamma$, i.e. $N_{i} \Delta_{i}=L_{i}$ holds for $i=x, y, z$. For simplicity, we assume the sampling parameters are the same along the $x$ and $y$ directions: $N_{x}=N_{y}$, $\Delta_{x}=\Delta_{y}$, and $L_{x}=L_{y}=N_{x} \Delta_{x}$. The wavenumber is sampled uniformly over the interval [ $\left.k_{\min }, k_{\max }\right]$ with sampling interval $\Delta_{k}$; the $i$-th measurement wavenumber is $k_{i} \triangleq k_{\min }+i \Delta_{k}$. We acquire data at $N_{f}$ focal planes, written $\left\{z_{i}: i=1,2, \ldots N_{f}\right\}$. The same sampling parameters are used at each focal plane; in particular, the set of sampled wavenumbers does not change.

We choose the sampling parameters as we would for a standard, single-species ISAM problem. The necessary sampling intervals can be motivated using the approximate forward model (4). Under this model, it can be shown that "point spread function" $|A(\boldsymbol{\rho}, k, z)|$ (approximately) decays like a Gaussian in $|\boldsymbol{\rho}|$; the measurements are "essentially" space limited 28. We take $L_{x}$ large enough to safely neglect the unmeasured data. Moreover, for fixed $z_{f}$, the measurements are bandlimited in q to [- $\left.k_{\max } \sin N A, k_{\max } \sin \mathrm{NA}\right]$; we sample along the transverse dimension at intervals $\Delta_{x}, \Delta_{y}<\pi /\left(k_{\max } \sin \mathrm{NA}\right)$.

\subsection{Discretization}

Given samples of the measurements [1), we take the 2D Discrete Fourier Transform (DFT) with respect to the transverse coordinates and write the result as the array $\hat{\mathbf{S}} \in \mathbb{C}^{N_{x} \times N_{x} \times N_{k} \times N_{f}}$. For simplicity, we take $N_{x}$ to be even. The 2D-DFT coordinate is written $\mathbf{q} \in\left[N_{x}\right]^{2}$. 


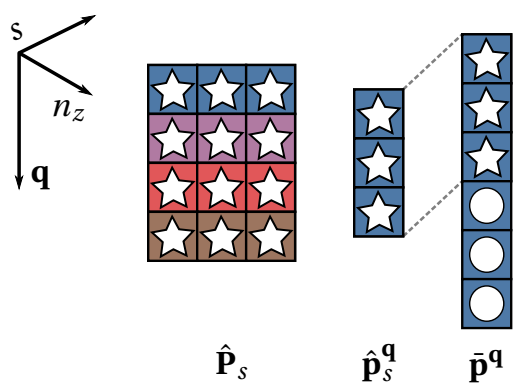

Figure 3: The various unfoldings of the discretized spatial densities with $N_{s}=2$. Here, block color indicates the value of q. Species 1 is marked with a star, while species 2 is indicated with a circle.

As the measurements are bandlimited and the object is compactly supported, the DFT of the measurements can be well-approximated by samples of the Fourier transform of the continuous model 2] 27. The discretized $N$-species measurement model is

$$
\hat{\mathbf{S}}[\mathbf{q}, m, f]=\sum_{s=1}^{N_{s}} \mathbf{h}_{s}[m] \sum_{n=0}^{N_{z}-1} \hat{\mathbf{A}}_{f}[\mathbf{q}, m, n] \hat{\mathbf{P}}_{s}[\mathbf{q}, n],
$$

where $\mathbf{h}_{s} \in \mathbb{C}^{N_{k}}$ and $\hat{\mathbf{P}}_{s} \in \mathbb{C}^{N_{x} \times N_{x} \times N_{z}}$ are the discretized spectral profile and the 2D-DFT of the $s$-th spatial density with respect to the transverse coordinates. The coefficients $\hat{\mathbf{A}}_{f}$ are obtained by sampling the continuous ISAM kernel [3]; that is,

$$
\hat{\mathbf{A}}_{f}[\mathbf{q}, m, n] \triangleq \hat{A}\left(\boldsymbol{\kappa}(\mathbf{q}), k_{\min }+m \Delta_{k}, n \Delta_{z}-z_{f}\right) .
$$

Here, $\boldsymbol{\kappa}(\mathbf{q}) \triangleq\left(k_{x}\left(q_{x}\right), k_{y}\left(q_{y}\right)\right)$ relates the DFT index $\mathbf{q}$ to the continuous Fourier coordinate $\boldsymbol{\kappa}$, where

$$
k_{x}\left(q_{x}\right)= \begin{cases}2 \pi q_{x} / L_{x} & q_{x}<N_{x} / 2 \\ 2 \pi\left(q_{x}-N_{x}\right) / L_{x} & \text { otherwise }\end{cases}
$$

and the same holds for $q_{y}$ and $k_{y}$.

\subsection{Block-Matrix Form of $N$-Species Forward Model}

With the spectral profiles fixed, the measurements $\hat{\mathbf{S}}$ are a linear function of the spatial densities. We can write the forward model [8] as a matrix-vector product, where the vector depends only on the spatial densities. Moreover, as the forward model is separable in $\mathbf{q}$, the resulting matrix has a block-diagonal structure. In what follows, we will analyze the inverse problem for each $\mathbf{q}$ independently.

We write the forward model [8] as a separate linear system for each q. Recall upper-case bold letters refer to matrices or arrays and lower-case bold letters refer to vectors. We use a bar to denote objects that have been "stacked" or vectorized. Subscripts are used to slice a array with respect to the last index: e.g., $\hat{\mathbf{S}}_{f}$ represents all measurements from the $f$-th focal plane, while $\mathbf{h}_{s}$ is the spectral profile for the $s$-th species. A superscript indicates a submatrix or vector formed for particular value of $\mathbf{q}$. In particular, we define

$$
\begin{aligned}
& \hat{\mathbf{s}}_{f}^{\mathbf{q}} \triangleq \hat{\mathbf{S}}[\mathbf{q},:, f] \in \mathbb{C}^{N_{k}} \\
& \hat{\mathbf{A}}_{f}^{\mathbf{q}} \triangleq \hat{\mathbf{A}}_{f}[\mathbf{q},:,:] \in \mathbb{C}^{N_{k} \times N_{z}} \\
& \hat{\mathbf{p}}_{s}^{\mathbf{q}} \triangleq \hat{\mathbf{P}}_{s}[\mathbf{q},:] \in \mathbb{C}^{N_{z}} .
\end{aligned}
$$

Further, define the diagonal matrix $\mathbf{D}_{s} \triangleq \operatorname{diag}\left(\mathbf{h}_{s}\right) \in \mathbb{C}^{N_{k} \times N_{k}}$. Now, for fixed $\mathbf{q}$ and $f$, 8) can be written

$$
\hat{\mathbf{s}}_{f}^{\mathbf{q}}=\sum_{s=1}^{N_{s}} \mathbf{D}_{s} \hat{\mathbf{A}}_{f}^{\mathbf{q}} \hat{\mathbf{p}}_{s}^{\mathbf{q}} .
$$


The collection of $[11]$ for $f \in\left[N_{f}\right]$ can be written as a single linear system in block form as

$$
\left[\begin{array}{c}
\hat{\mathbf{s}}_{1}^{\mathbf{q}} \\
\vdots \\
\hat{\mathbf{s}}_{N_{f}}^{\mathbf{q}}
\end{array}\right]=\left[\begin{array}{ccc}
\mathbf{D}_{1} \hat{\mathbf{A}}_{1}^{\mathbf{q}} & \ldots & \mathbf{D}_{N_{s}} \hat{\mathbf{A}}_{1}^{\mathbf{q}} \\
\vdots & \ddots & \vdots \\
\mathbf{D}_{1} \hat{\mathbf{A}}_{N_{f}}^{\mathbf{q}} & \cdots & \mathbf{D}_{N_{s}} \hat{\mathbf{A}}_{N_{f}}^{\mathbf{q}}
\end{array}\right]\left[\begin{array}{c}
\hat{\mathbf{p}}_{1}^{\mathbf{q}} \\
\vdots \\
\hat{\mathbf{p}}_{N_{s}}^{\mathbf{q}}
\end{array}\right],
$$

or concisely as

$$
\overline{\mathbf{s}}^{\mathbf{q}}=\Phi^{\mathbf{q}} \overline{\mathbf{p}}^{\mathbf{q}}
$$

where the vectors

$$
\begin{gathered}
\overline{\mathbf{p}}^{\mathbf{q} \triangleq} \operatorname{vec}(\hat{\mathbf{P}}[\mathbf{q},:,:])=\left[\left(\hat{\mathbf{p}}_{1}^{\mathbf{q}}\right)^{\top}, \ldots,\left(\hat{\mathbf{p}}_{N_{s}}^{\mathbf{q}}\right)^{\top}\right]^{\top} \in \mathbb{C}^{N_{s} N_{z}} \\
\overline{\mathbf{s}}^{\mathbf{q} \triangleq} \operatorname{vec}(\hat{\mathbf{S}}[\mathbf{q},:,:])=\left[\left(\hat{\mathbf{s}}_{1}^{\mathbf{q}}\right)^{\top}, \ldots,\left(\hat{\mathbf{s}}_{N_{f}}^{\mathbf{q}}\right)^{\top}\right]^{\top} \in \mathbb{C}^{N_{f} N_{k}},
\end{gathered}
$$

contain the spatial densities for each species and measurement for all focal planes, respectively, and

$$
\Phi^{\mathbf{q}} \triangleq\left[\begin{array}{ccc}
\mathbf{D}_{1} \hat{\mathbf{A}}_{1}^{\mathbf{q}} & \ldots & \mathbf{D}_{N_{s}} \hat{\mathbf{A}}_{1}^{\mathbf{q}} \\
\vdots & \ddots & \vdots \\
\mathbf{D}_{1} \hat{\mathbf{A}}_{N_{f}}^{\mathbf{q}} & \cdots & \mathbf{D}_{N_{s}} \hat{\mathbf{A}}_{N_{f}}^{\mathbf{q}}
\end{array}\right] \in \mathbb{C}^{N_{f} N_{k} \times N_{s} N_{z}} .
$$

Each block-row of $\Phi^{\mathbf{q}}$ corresponds to a single transverse Fourier component of measurements taken at a single focal plane, and the $s$-th block-column corresponds to the $s$-th species.

\subsection{Construction using Khatri-Rao product}

We briefly discuss an alternative construction of $\Phi^{\mathbf{q}}$ that connects the $N$-species inverse problem to a broad range of related problems, which we explore in Section 5.2.3.

Definition 2. The row-wise Khatri-Rao product of matrices $\mathbf{A} \in \mathbb{C}^{m \times n_{1}}$ and $\mathbf{B} \in \mathbb{C}^{m \times n_{2}}$ is

$$
\mathbf{A} \odot \mathbf{B}=\left[\begin{array}{c}
\mathbf{A}[1,:] \otimes \mathbf{B}[1,:] \\
\vdots \\
\mathbf{A}[m,:] \otimes \mathbf{B}[m,:]
\end{array}\right] \in \mathbb{C}^{m \times n_{1} n_{2}},
$$

i.e. each row of $\mathbf{A} \odot \mathbf{B}$ is the Kronecker product of the corresponding rows of $\mathbf{A}$ and $\mathbf{B}$.

We use the Khatri-Rao product to construct $\Phi^{\mathbf{q}}$. First, we define the matrix of spectral profiles $\mathbf{H} \in \mathbb{C}^{N_{k} \times N_{s}}$ by

$$
\mathbf{H}[m, s]=\mathbf{h}_{s}[m] .
$$

Now, the first block-row of $\Phi^{\mathbf{q}}$ is $\mathbf{H} \odot \hat{\mathbf{A}}_{1}^{\mathbf{q}}$. To obtain all block-rows of $\Phi^{\mathbf{q}}$, we first stack the $\left\{\hat{\mathbf{A}}_{f}^{\mathbf{q}}\right\}_{f=1}^{N_{f}}$ into the matrix $\overline{\mathbf{A}} \mathbf{q} \triangleq\left[\left(\hat{\mathbf{A}}_{1}^{\mathbf{q}}\right)^{\top} \ldots\left(\hat{\mathbf{A}}_{N_{f}}^{\mathbf{q}}\right)^{\top}\right]^{\top} \in \mathbb{C}^{N_{f} N_{k} \times N_{z}}$. Next, stack $N_{f}$ copies of $\mathbf{H}$ into $\overline{\mathbf{H}} \triangleq\left(\mathbb{1}_{N_{f}}^{\top} \otimes \mathbf{H}\right)=\left[\mathbf{H}^{\top}, \ldots, \mathbf{H}^{\top}\right]^{\top} \in \mathbb{C}^{N_{f} N_{k} \times N_{s}}$. Now, $\Phi^{\mathbf{q}}=\overline{\mathbf{H}} \odot \overline{\mathbf{A}}^{\mathbf{q}}$.

\section{The $N$-Species Inverse Problem}

\subsection{Preliminaries: The Single Species Case}

Under the $\mathrm{N}$-species model (8), the measurements at each focal plane are modeled as the sum of $N_{s}$ independent ISAM experiments; thus, the ISAM matrices $\hat{\mathbf{A}}_{f}^{\mathbf{q}}$ set fundamental limits on what can be imaged. Stated plainly, if a spatial density lies in the null space of each $\hat{\mathbf{A}}_{f}^{\mathbf{q}}$, then it will generate no measurement and thus cannot be imaged using the proposed method.

A careful study of the spectral properties of these matrices is beyond the scope of this paper. Instead, we combine a numerical study of these matrices with intuition obtained from the approximate ISAM kernel (4). We computed the singular values of $\hat{\mathbf{A}}_{f}^{\mathbf{q}}$ in the case of one transverse dimension, $x$, using the computational parameters 

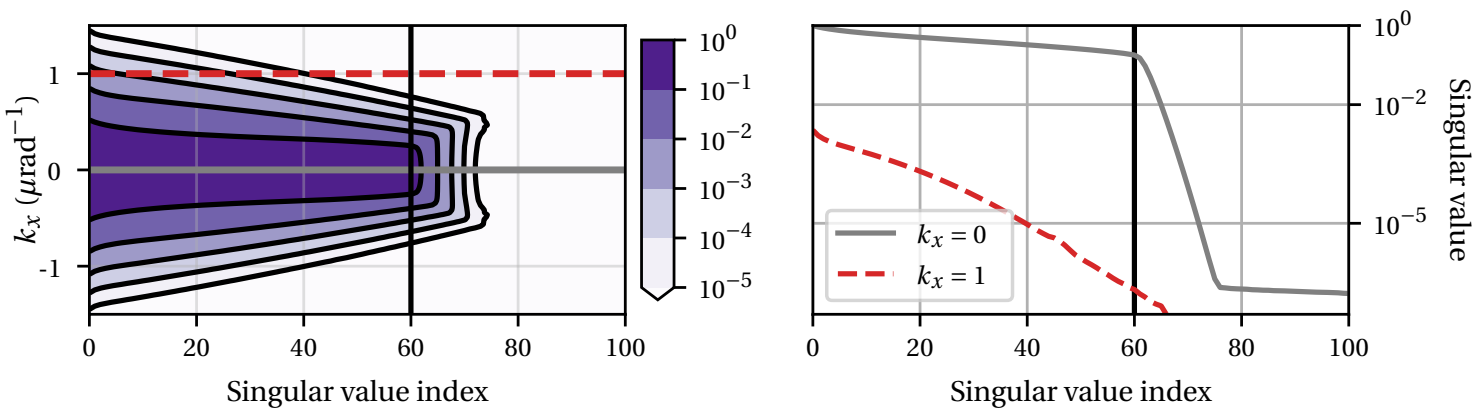

Figure 4: Left: Singular values of $\hat{\mathbf{A}}_{f}^{q_{x}}$ in the case of one transverse dimension. The coordinate $k_{x}$ is obtained from $q_{x}$ using (10). Right: singular values for $k_{x}=0$ and $k_{x}=1$. The vertical line marks the rank estimate (13). The focal plane is located at $z_{f}=140 \mu \mathrm{m}$. The remaining system parameters are listed in Table 1

listed in Table 1 . The singular values are shown in Fig. 4 where the continuous Fourier coordinate $k_{x}$ is determined from the DFT index $q_{x}$ using 10 . While we do not form $\hat{\mathbf{A}}_{f}^{\mathbf{q}}$ using the approximate kernel, the approximate kernel provides intuition for the behavior seen here. The largest singular values die off quickly as $k_{x}$ increases, as expected due to the function $\vartheta(\boldsymbol{\kappa}, k)$ in (4). Moreover, for $\left|k_{x}\right|>2 k_{\max }$, ISAM matrix is uniformly zero due to $\chi(\boldsymbol{\kappa}, 2 k)$.

According to the approximate forward model (5), for $k_{x}=0$ we obtain the (bandlimited) Fourier transform of the space-limited weighted susceptibility. The eigenvalue spectrum of space-and-frequency limited Fourier operators has been studied, beginning with a series of papers by Slepian, Landau, and Pollak [28-32. In the discrete case, the eigenvalue and singular value spectrum of space-and-frequency limited Discrete Fourier Transform (DFT) matrices have been studied; such matrices are submatrices formed by consecutive rows and columns of a DFT matrix [33-35]. The singular values of a space-and-frequency limited DFT matrix are divided into three distinct regions: (1) a region wherein the singular values are near one; (2) a transition region where the singular values decay exponentially; and (3) the remaining singular values are nearly zero. The number of singular values in the first region is called the effective rank and is written $r_{e}$. A direct application of Slepian-Pollak theory predicts 31 35]

$$
r_{e}=\frac{2\left(k_{\max }-k_{\min }\right)}{2 \pi / L_{z}}=\frac{L_{z}}{\pi}\left(k_{\max }-k_{\min }\right)
$$

For fixed $\boldsymbol{\kappa}$, the approximate ISAM operator can be viewed as a space-and-frequency limited Fourier operator with additional weighting in the spatial domain by $v(z)$ and in the frequency domain by $\vartheta(\boldsymbol{\kappa}, k)$. For each $\boldsymbol{\kappa}$ the operator is space-limited to a region of length $L_{z}$; this is due to assumption that $\eta$ is compactly supported. Moreover, the operator is frequency-limited to the optical passband B. In the discretized setting, only $\mathbf{A}_{f}^{0}$ can be viewed as a (diagonally scaled) DFT matrix, as for $\mathbf{q} \neq 0$ the resulting Fourier transform is not uniformly sampled.

We can use the theory of space-and-frequency limited DFT matrices to understand the behavior of the spectrum of $\hat{\mathbf{A}}_{f}^{0}$ as shown in Fig. 4 The singular values are broken into three regions: in the first region, the singular values decay exponentially, albeit at a rate slower than in the second region. The transition between the first and second regions still occurs at $r_{e}$. In the case of the parameters used in Fig. 4 we have $r_{e}=60$, and the change in behavior at $r_{e}$ is evident. The case of $k_{x} \neq 0$ is more complicated as the resulting Fourier transform is not uniformly sampled.

Recall that B is the set of observable Fourier components of the weighted susceptibility, $v\left(z-z_{f}\right) \hat{p}(\boldsymbol{\kappa}, z)$. A common practice in ISAM imaging is to ignore the axial weighting function and treat $B$ as the observable Fourier components of the unweighted susceptibility (see, e.g. [9] 11]). This is a reasonable approximation of the imaging system. To justify the approximation, note that $v(z)$ is strictly positive and slowly varying; thus the Fourier transform of the weighted and unweighted susceptibilities are roughly supported on the same set.

Using the same line of reasoning, we assume that null $\left\{\hat{\mathbf{A}}_{f}^{\mathbf{q}}\right\}$ is invariant to the choice of focal plane $z_{f}$. This is reasonable when the focal planes are close to one another. Note that this is an implicit assumption in previous work on multi-focal ISAM [36]. 


\subsection{Algebraic Conditions for a Unique Solution to (P1)}

We now consider the discretized $N$-species inverse problem. We begin by considering the discretized form of (P1) we assume the spectral profiles $\mathbf{h}_{s}$ are fixed and known. In this case, recovery of the spatial densities from measurements of the form $\left[8\right.$ is a linear inverse problem. We study the each "one-dimensional" problem $\overline{\mathbf{s}}^{\mathbf{q}}=\Phi^{\mathbf{q}} \overline{\mathbf{p}}^{\mathbf{q}}$ for $\mathbf{q} \in\left[N_{x}\right]^{2}$, with $\Phi^{\mathbf{q}}$ given by 12 . In the remainder of this section the DFT index $\mathbf{q}$ is fixed.

Without additional constraints on the spatial densities, the existence and uniqueness of a solution is determined entirely by the matrices $\Phi^{\mathbf{q}}$. In this section, we establish algebraic conditions for existence and uniqueness of a solution in terms of the ISAM matrices, $\left\{\hat{\mathbf{A}}_{f}^{\mathbf{q}}\right\}_{f=1}^{N_{f}}$, and the chemical spectra, $\left\{\mathbf{h}_{s}\right\}_{s=1}^{N_{s}}$. Earlier work on this problem claimed that $N_{f} \geq N_{s}$ and linear independence of the $\mathbf{h}_{s}$ is necessary and sufficient for unique recovery of the spatial densities within the optical passband [15]. While necessary, we show these two conditions are not sufficient.

For each focal plane, the ISAM matrix $\hat{\mathbf{A}}_{f}^{\mathbf{q}}$ is of size $N_{k} \times N_{z}$, where $N_{k}$ is the number of wavenumber samples and $N_{z}$ is the (axial) length of the discretized spatial density. Per Section 5.1 we assume the null space of $\hat{\mathbf{A}}_{f}^{\mathbf{q}}$ is invariant to the choice of focal plane, thus for fixed $\mathbf{q}$ each matrix has the same rank. Let $r \triangleq \operatorname{rank}\left(\hat{\mathbf{A}}_{f}^{\mathbf{q}}\right)$ for $f \in\left[N_{f}\right]$. We write the shared nullspace of the ISAM matrices as $\mathrm{N}^{\mathbf{q}} \subseteq \mathbb{C}^{N_{z}}$; we have

$$
\mathrm{N}^{\mathbf{q}} \triangleq \operatorname{null}\left\{\hat{\mathbf{A}}_{f}^{\mathbf{q}}\right\} \text { for } f \in\left[N_{f}\right] .
$$

The optical passband is $\left(\mathrm{N}^{\mathbf{q}}\right)^{\perp}$. Define the subspace

$$
\overline{\mathrm{N}}^{\mathbf{q}} \triangleq \mathrm{N}^{\mathbf{q}} \times \mathrm{N}^{\mathbf{q}} \ldots \times \mathrm{N}^{\mathbf{q}}=\operatorname{span}\left\{\overline{\mathbf{p}}^{\mathbf{q}}=\left[\left(\hat{\mathbf{p}}_{1}^{\mathbf{q}}\right)^{\top}, \ldots,\left(\hat{\mathbf{p}}_{N_{s}}^{\mathbf{q}}\right)^{\mathrm{T}}\right]^{\mathrm{T}} \mid \hat{\mathbf{p}}_{s}^{\mathbf{q}} \in \mathrm{N}^{\mathbf{q}}, s \in\left[N_{s}\right]\right\} \subseteq \mathbb{C}^{N_{s} N_{z}}
$$

of block vectors where each block is in $N^{\mathbf{q}}$. The subspace $(\bar{N} \mathbf{q})^{\perp}$ consists of block vectors where each block lies in the optical passband, $(\mathrm{N} \mathbf{q})^{\perp}$. In an abuse of notation, we refer to both $(\mathrm{N} \mathbf{q})^{\perp}$ and $(\overline{\mathrm{N}} \mathbf{q})^{\perp}$ as "the optical passband".

Using the $N$-species model, the measurements are a weighted sum of ISAM experiments; thus any objects that lie in $\bar{N}^{\mathbf{q}}$ will also be in null $\left\{\Phi^{\mathbf{q}}\right\}$. If an object cannot be imaged using ISAM, it cannot be imaged using $\Phi^{\mathbf{q}}$. We must consider uniqueness modulo $\overline{\mathrm{N}}^{\mathbf{q}}$; we wish to establish conditions such that these are the only objects that cannot be imaged using $\Phi^{\mathbf{q}}$. In this case, the $N$-species model does not introduce additional ambiguity and each species is correctly identified. We do no worse using the $N$-species model than if we were able to image the spatial densities independently using the ISAM system.

Let us pause to consider the geometry of a simple case: two species and a single focal plane. Here, $\Phi^{\mathbf{q}}=$ $\left[\mathbf{D}_{1} \hat{\mathbf{A}}_{1}^{\mathbf{q}}, \mathbf{D}_{2} \hat{\mathbf{A}}_{1}^{\mathbf{q}}\right]$ and $\overline{\mathbf{s}}^{\mathbf{q}}=\Phi^{\mathbf{q}} \overline{\mathbf{p}}^{\mathbf{q}}=\mathbf{D}_{1} \hat{\mathbf{A}}_{1}^{\mathbf{q}} \hat{\mathbf{p}}_{1}+\mathbf{D}_{2} \hat{\mathbf{A}}_{1}^{\mathbf{q}} \hat{\mathbf{p}}_{2}$. Clearly, if $\hat{\mathbf{p}}_{1}^{\mathbf{q}}$ and $\hat{\mathbf{p}}_{2}^{\mathbf{q}}$ are each in $\mathbf{N} \mathbf{q}$, then $\overline{\mathbf{s}}^{\mathbf{q}}=\mathbf{0}$. Suppose the $\mathbf{h}_{s}$ are non-zero for each index; then $\mathbf{D}_{s}$ is full rank. Using the formula for the rank of a partitioned matrix,

$$
\begin{aligned}
\operatorname{rank}\left(\Phi^{\mathbf{q}}\right)=\operatorname{rank}\left(\left[\mathbf{D}_{1} \hat{\mathbf{A}}_{1}^{\mathbf{q}}, \mathbf{D}_{2} \hat{\mathbf{A}}_{1}^{\mathbf{q}}\right]\right) & =\operatorname{rank}\left(\mathbf{D}_{1} \hat{\mathbf{A}}_{1}^{\mathbf{q}}\right)+\operatorname{rank}\left(\mathbf{D}_{2} \hat{\mathbf{A}}_{1}^{\mathbf{q}}\right)-\operatorname{dim}\left(\operatorname{range}\left\{\mathbf{D}_{1} \hat{\mathbf{A}}_{1}^{\mathbf{q}}\right\} \cap \operatorname{range}\left\{\mathbf{D}_{2} \hat{\mathbf{A}}_{1}^{\mathbf{q}}\right\}\right) \\
& =2 r-\operatorname{dim}\left(\operatorname{range}\left\{\mathbf{D}_{1} \hat{\mathbf{A}}_{1}^{\mathbf{q}}\right\} \cap \operatorname{range}\left\{\mathbf{D}_{2} \hat{\mathbf{A}}_{1}^{\mathbf{q}}\right\}\right) .
\end{aligned}
$$

The last term captures the interplay between the $\mathbf{D}_{s}$ and $\hat{\mathbf{A}}_{1}^{\mathbf{q}}$. We want to find conditions under which this intersection is trivial. As we assume $\mathbf{D}_{s}$ is full rank, we can instead ask when range $\left\{\hat{\mathbf{A}}_{1}^{\mathbf{q}}\right\} \cap$ range $\left\{\mathbf{D}_{1}^{-1} \mathbf{D}_{2} \hat{\mathbf{A}}_{1}^{\mathbf{q}}\right\}$ is trivial. Loosely speaking, when is multiplication by a diagonal matrix enough to perturb a subspace out of alignment with itself?

Next, we define a notion of uniqueness modulo the ISAM nullspace.

Definition 3. The solution to $\overline{\mathbf{s}}^{\mathbf{q}}=\Phi^{\mathbf{q}} \overline{\mathbf{p}}^{\mathbf{q}}$ is said to be unique within the optical passband if $\Phi^{\mathbf{q}} \mathbf{x}=\Phi^{\mathbf{q}} \mathbf{y} \Longrightarrow \mathbf{x}-\mathbf{y} \in \overline{\mathrm{N}} \mathbf{q}$. Equivalently, there is a unique $\overline{\mathbf{p}}^{\mathbf{q}} \in(\bar{N} \mathbf{q})^{\perp}$ such that $\overline{\mathbf{s}}^{\mathbf{q}}=\Phi^{\mathbf{q}} \overline{\mathbf{p}}^{\mathbf{q}}$.

This definition sets up an equivalence relation on the spatial densities: we treat two spatial densities as equivalent if their difference lies in $\bar{N} \mathbf{q}$, the null space of the ISAM matrices. This is the component to which we are inherently are blind even in the single species case.

Next, we cast the problem into a form where we implicitly work in the optical passband $(\overline{\mathrm{N}} \mathbf{q})^{\perp}$. Let $\mathbf{V}^{\mathbf{q}} \in \mathbb{C}^{N_{z} \times r}$ be a basis for $\left(\mathrm{N}^{\mathbf{q}}\right)^{\perp}$. We introduce a new set of matrices: the restricted ISAM matrix $\hat{\mathbf{B}}_{f}^{\mathbf{q}} \triangleq \hat{\mathbf{A}}_{f}^{\mathbf{q}} \mathbf{V}^{\mathbf{q}} \in \mathbb{C}^{N_{k} \times r}$ is the restriction of $\hat{\mathbf{A}}_{f}^{\mathbf{q}}$ to the subspace $(\mathrm{N} \mathbf{q})^{\perp}$. Clearly, $\hat{\mathbf{B}}_{f}^{\mathbf{q}}$ has full column rank. Similarly, $\mathbf{I}_{N_{f}} \otimes \mathbf{V}^{\mathbf{q}}$ is a basis for $(\overline{\mathrm{N}} \mathbf{q})^{\perp}$. We define the restricted $N$-species matrix

$$
\left.\tilde{\Phi}^{\mathbf{q}} \triangleq \Phi^{\mathbf{q}_{(\mathbf{I}}} \text { N }_{f} \mathbf{V}^{\mathbf{q}}\right) \in \mathbb{C}^{N_{f} N_{k} \times N_{s} r}
$$




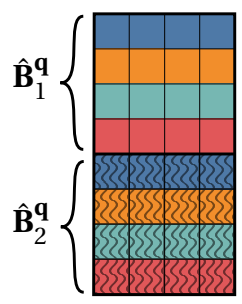

(a)

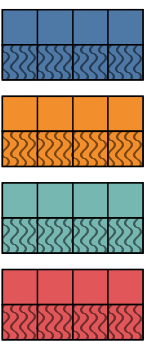

(b)
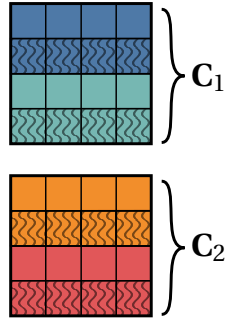

(c)

Figure 5: Comparing (N5) and Theorem 2 for $N_{f}=2$ and $r=4$. (a) The matrix $\overline{\mathbf{B}}^{\mathbf{q}}$. Color denotes the value of $k$. Rows with solid (resp. wave-patterned) blocks correspond to measurements at the first (resp. second) focal plane. (b) Condition (N5) requires that the sum of the ranks of each $2 \times 4$ block of the same color must be at least $4 N_{s}$. (c) A possible partitioning of the rows of $\overline{\mathbf{B}}^{\mathbf{q}}$ as described in Theorem 2 . If both $\mathbf{C}_{1}$ and $\mathbf{C}_{2}$, as defined in 15), have full rank for generic chemical species the solution to $\overline{\mathbf{s}}^{\mathbf{q}}=\Phi^{\mathbf{q}} \overline{\mathbf{p}}^{\mathbf{q}}$ is unique within the optical passband with probability one.

The question of unique recovery (within the optical passband) is determined entirely by this matrix, as stated in the following result.

Lemma 1. Let $\Phi^{\mathbf{q}} \in \mathbb{C}^{N_{f} N_{k} \times N_{s} N_{z}}$ and $\operatorname{rank}\left(\hat{\mathbf{A}}_{f}^{\mathbf{q}}\right)=r$ for $f \in\left[N_{f}\right]$. The following statements are equivalent:

(C1) There is a unique $\overline{\mathbf{p}}^{\mathbf{q}} \in(\overline{\mathrm{N}} \mathbf{q})^{\perp}$ such that $\overline{\mathbf{s}}^{\mathbf{q}}=\Phi^{\mathbf{q}} \overline{\mathbf{p}}^{\mathbf{q}}$

(C2) $\operatorname{null}\left\{\Phi^{\mathbf{q}}\right\}=\bar{N}^{\mathbf{q}}$

(C3) $\operatorname{rank}\left(\tilde{\Phi}^{\mathbf{q}}\right)=N_{s} r$.

We defer the proof to Appendix A

We can construct the restricted $N$-species matrix $\tilde{\Phi}^{\mathbf{q}}$ using the Khatri-Rao product. Let $\overline{\mathbf{B}}^{\mathbf{q}} \in \mathbb{C}^{N_{f} N_{k} \times r}$ be the matrix formed by stacking the restricted ISAM matrices $\hat{\mathbf{B}}_{f}^{\mathbf{q}}$ into a single block column: $\overline{\mathbf{B}}^{\mathbf{q}} \triangleq\left[\left(\hat{\mathbf{B}}_{1}^{\mathbf{q}}\right)^{\top}, \ldots,\left(\hat{\mathbf{B}}_{N_{f}}^{\mathbf{q}}\right)^{\top}\right]^{\top}$. Recall $\overline{\mathbf{H}}=\left(\mathbb{1}_{N_{f}}^{\top} \otimes \mathbf{H}\right)=\left[\mathbf{H}^{\top}, \ldots, \mathbf{H}^{\top}\right] \in \mathbb{C}^{N_{f} N_{k} \times N_{s}}$. Now,

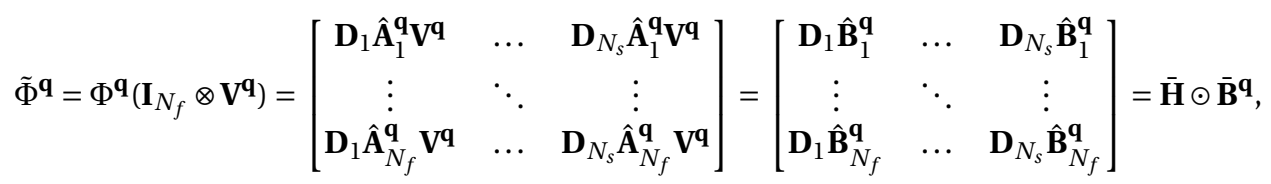

mirroring the construction of $\Phi^{\mathbf{q}}$ in Section 4.4

In what follows, we establish necessary and sufficient conditions for uniqueness within the optical passband.

\subsubsection{Necessary Conditions for Uniqueness}

Theorem 1. The solution to $\overline{\mathbf{s}}^{\mathbf{q}}=\Phi^{\mathbf{q}} \overline{\mathbf{p}}^{\mathbf{q}}$ is unique within the optical passband only if

(N1) $N_{k} N_{f} \geq N_{s} r$

(N2) The spectral profiles are linearly independent $\left(\operatorname{rank}(\mathbf{H})=N_{s}\right)$

(N3) No row of $\overline{\mathbf{B}}^{\mathbf{q}}$ is orthogonal to all remaining rows

(N4) For every subset $J \subset\left[N_{k}\right]$ with $N_{s} \leq|J|<N_{s} r / N_{f}$ and $\operatorname{rank}\left(\mathbf{H}^{J}\right)=N_{s}$, we have $\operatorname{rank}\left(\mathbf{H}^{J^{c}}\right) \geq N_{s}-\frac{N_{f}}{r}|J|$

(N5) $\sum_{i=1}^{N_{k}} \operatorname{rank}\left(\left[\hat{\mathbf{B}}_{1}^{\mathbf{q}}[i,:]^{\top}, \ldots, \hat{\mathbf{B}}_{N_{f}}^{\mathbf{q}}[i,:]^{\top}\right]^{\top}\right) \geq N_{s} r$. 
We defer the proof to Appendix A Let us pause to interpret these conditions.

In the single-species case, (N1) reduces to $N_{k} \geq r$; i.e. we must measure enough wavenumbers such that the single-species ISAM problem is well-posed. Interestingly, (N1) does not require that $N_{f} \geq N_{s}$ : recovery of $N_{s}$ species is possible from a single focal plane, provided the measurements are oversampled in wavenumber. This behavior can be seen in the numerical experiments described in Section 5.3

Condition (N2) is unsurprising. If the spectral profiles are linearly dependent, the $N$-species representation of a susceptibility is not unique and the spatial densities cannot be uniquely determined.

Condition (N3) is less transparent, but can be argued to hold by the underlying physics. If (N3) is violated, there must be an object that scatters at only one of the measured wavenumbers and is non-scattering for the rest. In the continuous setting, scattered fields are analytic functions of $k$; thus if an object is non-scattering over an interval of wavenumbers, it must be non-scattering for all $k$ [37, 38]. In the discretized setting we lose the analytic properties of scattered waves. In numerical simulations, however, condition (N3) holds.

Condition (N4) requires the spectral profiles to be sufficiently diverse: linear independence is not enough. As an example, consider $N_{s}=2, N_{f}=1$, and take $\mathbf{h}_{1}=[1,1, \ldots, 1]^{\top}$ and $\mathbf{h}_{2}=[2,1, \ldots, 1]^{\top}$. These spectra are linearly independent, but $\mathbf{D}_{1} \hat{\mathbf{A}}_{1}^{\mathbf{q}}$ and $\mathbf{D}_{2} \hat{\mathbf{A}}_{1}^{\mathbf{q}}$ differ by only one row; thus $\operatorname{rank}\left(\tilde{\Phi}^{\mathbf{q}}\right) \leq r+1$, failing $(\mathrm{C} 3)$ of Lemma 1 Spectral diversity is necessary to push range $\left\{\mathbf{D}_{1} \hat{\mathbf{A}}_{1}^{\mathbf{q}}\right\}$ out of alignment with range $\left\{\mathbf{D}_{2} \hat{\mathbf{A}}_{1}^{\mathbf{q}}\right\}$. "Good" spectral profiles are not too concentrated on any small set of indices.

The final condition, (N5), is a requirement on the diversity of measurements comprising the restricted ISAM matrices. When $N_{k} N_{f}=N_{s} r$, (N5) requires that the collection of measurement vectors corresponding to a given wavenumber be linearly independent: each new focal plane must provide new and informative measurements. This partitioning is illustrated in Fig.5.

\subsubsection{Sufficient Condition for Uniqueness}

First, we note that no conditions on $\overline{\mathbf{B}}^{\mathbf{q}}$ or $\mathbf{H}$ independently are sufficient to ensure there is a unique solution within the optical passband. Consider again the two-species, one focal plane case: $\tilde{\Phi}^{\mathbf{q}}=\left[\mathbf{D}_{1} \hat{\mathbf{B}}_{1}^{\mathbf{q}}, \mathbf{D}_{2} \hat{\mathbf{B}}_{1}^{\mathbf{q}}\right]$, with $\mathbf{D}_{i}=\operatorname{diag}\left(\mathbf{h}_{i}\right)$. Suppose $\mathbf{h}_{1}$ is fixed and choose vectors $\mathbf{w}, \mathbf{v} \in \mathbb{C}^{r}$ such that no entry of $\hat{\mathbf{B}}_{1}^{\mathbf{q}} \mathbf{v}$ is zero. Set $\mathbf{h}_{2}=\left(\mathbf{D}_{1} \hat{\mathbf{B}}_{1}^{\mathbf{q}} \mathbf{w}\right) /\left(\hat{\mathbf{B}}_{1}^{\mathbf{q}} \mathbf{v}\right)$ where the division is taken elementwise. With this construction, $\mathbf{D}_{2} \hat{\mathbf{B}}_{1}^{\mathbf{q}} \mathbf{v}=\mathbf{D}_{1} \hat{\mathbf{B}}_{1}^{\mathbf{q}} \mathbf{w}$, and thus $\operatorname{rank}\left(\tilde{\Phi}^{\mathbf{q}}\right) \leq 2 r-1$, failing (C3) of Lemma[1

These spectral profiles were carefully chosen to make $\tilde{\Phi}^{\mathbf{q}}$ lose rank. Fortunately, we are unlikely to encounter such objects in practice. The following definition makes this argument precise.

Definition 4. A property that depends on the spectral profiles $\mathbf{H} \in \mathbb{C}^{N_{k} \times N_{s}}$ is said to hold generically, or for generic $\mathbf{H}$, if the set for which it fails to hold has Lebesgue measure zero and is nowhere dense in $\mathbb{C}^{N_{k} \times N_{s}}$.

If a property that holds generically, it holds with probability one if the spectral profiles are drawn independently from a distribution that is absolutely continuous with respect to the Lebesgue measure in $\mathbb{C}^{N_{k} \times N_{s}}$; for instance, when the entries of $\mathbf{H}$ are drawn i.i.d. from the Gaussian distribution. Moreover, the property exhibits a degree of robustness: if it holds for a particular $\mathbf{H}^{\prime}$, then it holds in an open ball around $\mathbf{H}^{\prime}$ and will continue to hold given sufficiently small perturbations to $\mathbf{H}^{\prime}$.

Theorem 2. Suppose $N_{k} \geq r$ and $N_{f} \geq N_{s}$. If there exists a collection $\left\{J_{i} \subset\left[N_{k}\right]\right\}_{i=1}^{N_{f}}$ of disjoint sets, each of cardinality $\left|J_{i}\right|=r / N_{f}$, such that

$$
\mathbf{C}_{i} \triangleq\left[\begin{array}{c}
\hat{\mathbf{B}}_{1}^{\mathbf{q}}\left[J_{i},:\right] \\
\vdots \\
\hat{\mathbf{B}}_{N_{f}}^{\mathbf{q}}\left[J_{i},:\right]
\end{array}\right] \in \mathbb{C}^{r \times r}
$$

is full rank for each $i \in\left[N_{f}\right]$, then for generic $\mathbf{H}$ the solution to $\overline{\mathbf{s}}^{\mathbf{q}}=\Phi^{\mathbf{q}} \overline{\mathbf{p}}^{\mathbf{q}}$ is unique within the optical passband.

An illustration of the matrices $\mathbf{C}_{i}$ is shown in Fig. 5 (c). Note that the necessary condition(N5) coincides with the sufficient condition of Theorem 2 in the case of $N_{k}=N_{f}=r=N_{s}$, which is the limit of scanning confocal spectroscopic acquisition discussed in Section 1 .

Theorem 2 can be stated in terms of a more familiar, but more restrictive, property on $\overline{\mathbf{B}}^{\mathbf{q}}$.

Definition 5. The Kruskal (row) rank of a matrix $\mathbf{X} \in \mathbb{C}^{n \times m}$, written $\operatorname{krank}(\mathbf{X})$, is the largest $k$ such that every set of $k$ rows of $\mathbf{X}$ are linearly independent. The matrix $\mathbf{X}$ is said to have full Kruskal rank if $\operatorname{krank}(\mathbf{X})=\max \{n, m\}$. 
Corollary 1. If $\overline{\mathbf{B}}^{\mathbf{q}} \in \mathbb{C}^{N_{k} N_{f} \times r}$ has full Kruskal rank, then for generic $\mathbf{H}$ the solution to $\overline{\mathbf{s}}^{\mathbf{q}}=\Phi^{\mathbf{q}} \overline{\mathbf{p}}^{\mathbf{q}}$ is unique within the optical passband.

\subsubsection{Related Problems}

The Khatri-Rao structure of $\Phi^{\mathbf{q}}$ provides a link between the $N$-species inverse problem and topics in tensor factorization, communications, and sensor networks, among others [39-45]. For example, the rank and Kruskal rank of the Khatri-Rao product has implications for the uniqueness of certain tensor factorizations. Properties of the Khatri-Rao product are an active area of research. For generic matrices $\mathbf{X}$ and $\mathbf{Y}$, it is known that $\operatorname{krank}(\mathbf{X} \odot \mathbf{Y})=$ $\operatorname{krank}(\mathbf{X}) \operatorname{krank}(\mathbf{Y})$. Bhaskara et al. provide bounds on the smallest singular value of the Khatri-Rao product of random matrices 44 . Recent work has investigated the restricted isometry property of the Khatri-Rao product of random matrices $[40-42]$.

These results do not directly apply to the problem at hand. We are interested in properties of $\tilde{\Phi}^{\mathbf{q}}=\overline{\mathbf{H}} \odot \overline{\mathbf{B}}^{\mathbf{q}}$. As $\overline{\mathbf{B}}^{\mathbf{q}}$ is determined by the physics and imaging geometry, we cannot choose this matrix generically or randomly. Even $\overline{\mathbf{H}}$ cannot be chosen generically, as $\overline{\mathbf{H}}=\left(\mathbb{1}_{N_{f}}^{\top} \otimes \mathbf{H}\right)$; only the matrix $\mathbf{H}$ can be chosen generically. Translating new results on the Khatri-Rao product to our setting remains a topic for further investigation.

\subsection{Stability And Conditioning of (P1)}

The results of the previous section tell us that the solution to $\overline{\mathbf{s}}^{\mathbf{q}}=\Phi^{\mathbf{q}} \overline{\mathbf{p}}^{\mathbf{q}}$ is almost always unique (within the optical passband), but say little about the stability of the problem. We must always deal with "noisy" measurements- not just instrumentation noise, but also "noise" due to modeling error, e.g. multiple scattering and spatial-spectral coupling not captured by the $N$-species model.

In this section, we numerically investigate the behavior of the singular values of the $N$-species matrix $\Phi$ for the case three-species case $\left(N_{s}=3\right)$ in two spatial dimensions. We use the computational parameters listed in Table 1 . except for NA and $N_{f}$, which vary. The singular values of the ISAM matrix formed using these computational parameters were investigated in Section 5.1 and plotted in Fig. 4 The spectral profiles used-caffeine, acetaminophen, and warfarin - are shown in Fig. 8 .

We computed the singular values of each block-matrix $\Phi^{\mathbf{q}} 12$ and plot the results in Fig. 6 . Recall that the continuous Fourier frequency $k_{x}$ is determined from the DFT index $q_{x}$ using (10). As expected, higher transverse spatial frequencies are present as NA increases. Only the first $N_{f} r_{e}$ singular values are appreciable. The lowfrequency components achieve rank $3 r_{e}$ for $N_{f}=3$, and adding focal planes improves the conditioning of $\Phi$. Note that even in the case of a single focal plane, the $3 r_{e}$-th singular value of $\Phi^{0}$ is non-zero; as previously discussed, $N_{f} \geq N_{s}$ is not necessary for a unique solution.

We investigated the singular values of the block corresponding to $k_{x}=0$ for a variety of chemical species and a varying number of focal planes. We used a library of 20 experimentally acquired chemical spectra 1 provided through the IARPA SILMARILS project. We randomly selected three species from the library, formed $\Phi^{0}$, and computed the singular values of this matrix. We scaled $\Phi^{0}$ to have unit spectral norm. This procedure was repeated for 200 realizations. The resulting singular values are plotted in Fig. 7 the borders of the shaded region are the best and worst realizations for each choice of $N_{f}$.

We repeated the same procedure using random spectral profiles. The real part of the spectral profile was drawn i.i.d. from the standard normal distribution and the imaginary part was chosen uniformly over $[0,1]$. The results are plotted in Fig. 7. Clearly, these un-physical spectra lead to better conditioned $\Phi^{0}$, and there is little difference in the best and worst realizations. Study of the system using random spectral profiles may lead to a useful upper bound on system performance.

\subsection{Algebraic Conditions for (P2)}

We now focus on the case (P2) wherein the target comprises $N_{s}$ chemical species drawn from a "dictionary" of $M_{s}>N_{s}$ possible spectra. This problem can be viewed as an instance of (P1) in which case Theorem 1 requires that number of focal planes is chosen such that $N_{f} N_{k} \geq M_{s} r$. This is undesirable if $M_{s}$ is much larger than $N_{s}$.

\footnotetext{
${ }^{1}$ These include caffeine, acetaminophen, warfarin, monosodium glutamate (MSG), sucrose, naproxen, potassium chlorate, polyvinylidene fluoride (PVDF), aspartame, lactose, melatonin, ethylenediaminetetraacetic acid (EDTA), creatine, diazepam, biotin, fructose, pectin, glycine, beta carotene, hydroxypropyl cellulose.
} 


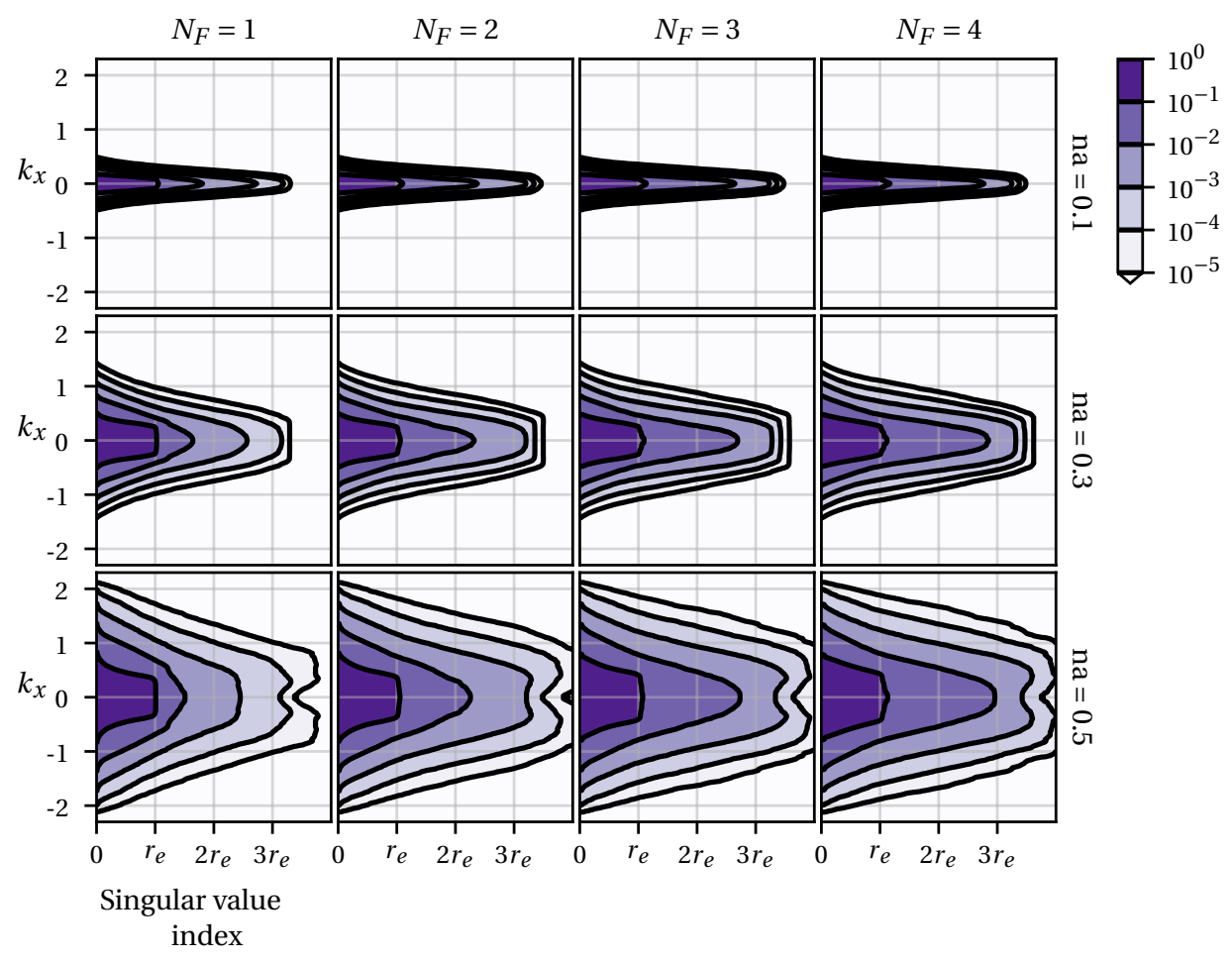

Figure 6: Singular values of $\Phi^{q}$ as a function of $k_{x}(q)$. Three species are present: caffeine, acetaminophen, and warfarin. System parameters listed in Table 1 .

This approach ignores the constraint that only $N_{s}$ chemicals are present in the sample; by incorporating this side information, we relax the condition on $N_{f}$. This structure is known as block sparsity.

Definition 6. The block vector $\overline{\mathbf{x}}=\left[\mathbf{x}_{1}^{\top}, \mathbf{x}_{2}^{\top}, \ldots, \mathbf{x}_{M_{s}}^{\top}\right]^{\top}$ is said to be block-Ksparse if the set $\left\{i:\left\|\mathbf{x}_{i}\right\|_{2}>0\right\}$ has cardinality at most $K$.

Block sparsity is a natural fit for this problem; we define the $s$-th block to be the $s$-th spatial density $\hat{\mathbf{p}}_{s}$, corresponding to the $s$-th species in the dictionary. Note that block sparsity does not require the blocks themselves (i.e., the $\left\{\hat{\mathbf{p}}_{s}\right\}_{s=1}^{N_{s}}$ ) to be sparse.

Conditions for unique recovery of block-sparse vectors have been studied [46-49]. Eldar and Mishali [48] developed a straightforward condition for unique recovery that is useful to the problem at hand:

Lemma 2. [48. Proposition 1] There is a unique block- $N_{s}$ sparse solution to $\overline{\mathbf{s}}^{\mathbf{q}}=\Phi^{\mathbf{q}} \overline{\mathbf{p}}^{\mathbf{q}}$ if and only if $\Phi^{\mathbf{q}} \mathbf{v} \neq 0$ for any non-zero $\mathbf{v}$ that is block- $2 N_{s}$ sparse.

We can easily translate Lemma 2 into this setting.

Theorem 3. For generic $\mathbf{H}$, within the optical passband there is a unique block- $N_{s}$ sparse vector $\overline{\mathbf{p}}^{\mathbf{q}}$ consistent with measurements $\overline{\mathbf{s}}^{\mathbf{q}}=\Phi^{\mathbf{q}} \overline{\mathbf{p}}^{\mathbf{q}}$ if $N_{k}>r, N_{f} \geq 2 N_{s}$, and $\overline{\mathbf{B}}^{\mathbf{q}}$ contains $2 N_{s}$ disjoint sets of linearly independent rows, each of cardinality $r=\operatorname{rank}\left(\overline{\mathbf{B}}^{\mathbf{q}}\right)$.

Proof. Let $\mathbf{v}$ be a block- $2 N_{s}$ sparse vector. Let $J=\left[j_{1}, \ldots, j_{2 N_{s}}\right]^{\top} \in \mathbb{Z}^{2 N_{s}}$ index the non-zero blocks of $\mathbf{v}$. The vector $\mathbf{v}_{J} \in \mathbb{C}^{2 N_{s} N_{z}}$ contains the non-zero elements of $\mathbf{v}$. The matrix $\Phi_{J}^{\mathbf{q}} \in \mathbb{C}^{N_{f} N_{k} \times 2 N_{s} N_{z}}$ is the restriction of $\Phi^{\mathbf{q}}$ to the $2 N_{s}$ columns indexed by $J$.

By assumption, $\overline{\mathbf{B}}^{\mathbf{q}}$ satisfies the conditions of Theorem 2 and $\Phi_{J}^{\mathbf{q}}$ is generically full column rank. Thus, for generic $\mathbf{H}$, we have $\Phi^{\mathbf{q}} \mathbf{v}=\Phi_{J}^{\mathbf{q}} \mathbf{v}_{J} \neq 0$. Applying Lemma 2 completes the proof. 

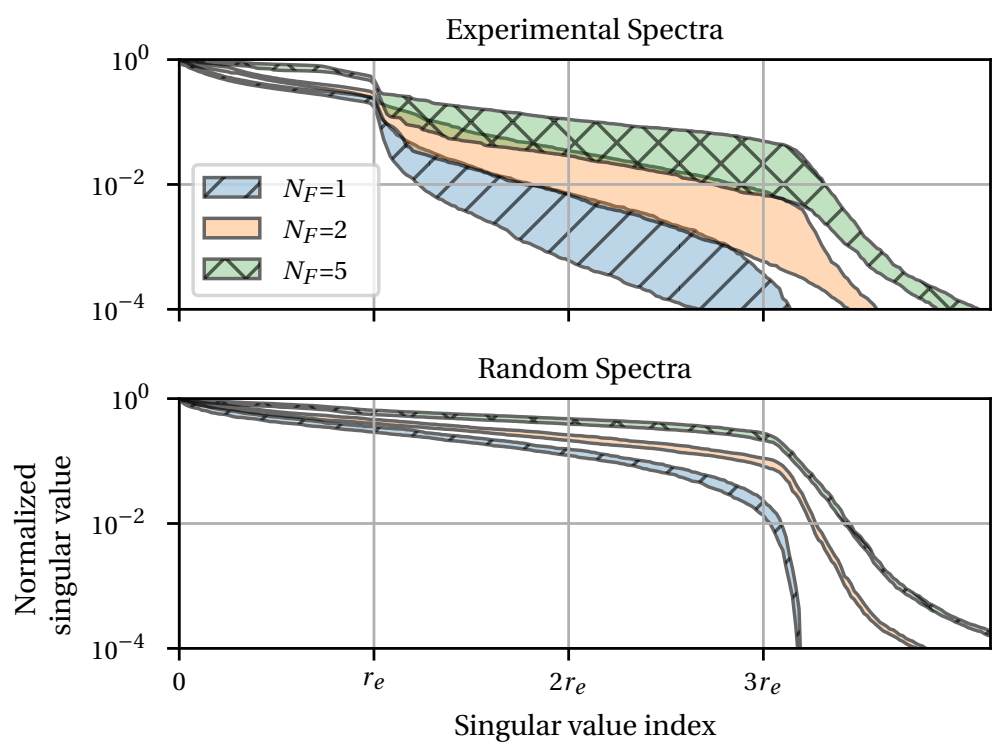

Figure 7: Singular values of $\Phi^{0}$ for various combinations of chemical species. The shaded area lies in between the best and worst realizations. System parameters listed in Table 1 Top: singular values using experimentally acquired spectral profiles. Bottom: singular values using random Gaussian spectral profiles.

\subsection{Computational Recovery}

In the single-species case, the approximate form of the ISAM operator (Section2.2 provides a non-iterative reconstruction based on Fourier resampling [50]. This does not carry over to the multi-species case and we must instead use an iterative approach.

To ease notation, define $\hat{\mathbf{p}}_{s} \triangleq \operatorname{vec}\left(\hat{\mathbf{P}}_{s}\right)$. We recover the collection of spatial densities by solving the penalized least squares problem

$$
\underset{\left\{\hat{\mathbf{p}}_{1}, \ldots, \hat{\mathbf{p}}_{N_{s}}\right\}}{\arg \min } \frac{1}{2} \sum_{\mathbf{q}}\left\|\overline{\mathbf{s}}^{\mathbf{q}}-\Phi^{\mathbf{q}} \overline{\mathbf{p}}^{\mathbf{q}}\right\|_{2}^{2}+R\left(\hat{\mathbf{p}}_{1}, \ldots, \hat{\mathbf{p}}_{N_{s}}\right) .
$$

The first term is known as the data fidelity term. It ensures the observed data and "re-imaged" solution are consistent. More sophisticated data fidelity terms can be used to model the effects of shot noise, background signal, and more [51], but these are beyond the scope of this work.

The functional $R: \mathbb{C}^{N_{s} \times N_{x} \times N_{x} \times N_{z}} \rightarrow \mathbb{R}$ regularizes the inverse problem and encodes any constraints or a priori assumptions regarding the spatial densities. Tikhonov regularization corresponds to $R\left(\hat{\mathbf{p}}_{1}, \ldots, \hat{\mathbf{p}}_{N_{s}}\right)=\sum_{s=1}^{N_{s}}\left\|\hat{\mathbf{p}}_{s}\right\|_{2}^{2}$. Alternatively, solutions that are sparse in a transform domain are obtained by setting $R\left(\hat{\mathbf{p}}_{1}, \ldots, \hat{\mathbf{p}}_{N_{s}}\right)=\sum_{s=1}^{N_{s}}\left\|\mathbf{C} \hat{\mathbf{p}}_{s}\right\|_{1}$. where $\mathbf{C}$ is a sparsifying transform, e.g. a wavelet transform. Finally, the mixed $\ell_{1} / \ell_{2}$ norm $\sum_{s=1}^{N_{s}}\left\|\hat{\mathbf{p}}_{s}\right\|_{2}$ encourages solutions that are block-sparse; that is, solutions with a minimal number of active species. The non-negative scalar $\lambda_{r}$ balances the influence of the data fidelity and regularization terms.

The method used to solve [16] depends on the chosen regularizer. In the case of Tikhonov regularization, (16) reduces to the solution of the linear system

$$
\left(\left(\Phi^{\mathbf{q}}\right)^{\mathrm{H}} \Phi^{\mathbf{q}}+\lambda_{r} \mathbf{I}\right) \overline{\mathbf{p}}^{\mathbf{q}}=\left(\Phi^{\mathbf{q}}\right)^{\mathrm{H}} \overline{\mathbf{s}}^{\mathbf{q}}
$$

for each $\mathbf{q} \in\left[N_{x}\right]^{2}$. The conjugate gradient algorithm works well in practice and requires only matrix-vector products with $\Phi^{\mathbf{q}}$ and $\left(\Phi^{\mathbf{q}}\right)^{\mathrm{H}}$. These matrices are not explicitly formed; only the coefficients $\hat{\mathbf{A}}_{f}^{\mathbf{q}}[\mathbf{q}, m, n]$ in $[9]$ are precomputed and stored. Similarly, the matrices $\mathbf{D}_{s}$ are not formed; only the spectral profiles are stored, and products with $\mathbf{D}_{s}$ are computed by elementwise multiplication. The vector $\overline{\mathbf{y}}^{\mathbf{q}}=\Phi^{\mathbf{q}} \overline{\mathbf{p}}^{\mathbf{q}}$ consists of blocks $\hat{\mathbf{y}}_{f}^{\mathbf{q}} \in \mathbb{C}^{N_{k}}$ for $f \in\left[N_{f}\right]$, with

$$
\hat{\mathbf{y}}_{f}^{\mathbf{q}}=\sum_{s=1}^{N_{s}} \mathbf{D}_{s} \hat{\mathbf{A}}_{f}^{\mathbf{q}} \hat{\mathbf{p}}_{s}^{\mathbf{q}}
$$




\begin{tabular}{|cc||cc||cc|}
\hline$N_{x}$ & 192 & $L_{x}$ & $423.6 \mu \mathrm{m}$ & $\Delta_{x}$ & $2.2 \mu \mathrm{m}$ \\
\hline$N_{z}$ & 384 & $L_{z}$ & $282.4 \mu \mathrm{m}$ & $\Delta_{z}$ & $0.7 \mu \mathrm{m}$ \\
\hline$N_{k}$ & 384 & $k_{\min }$ & $0.4 \mathrm{rad} \cdot \mu \mathrm{m}^{-1}$ & $k_{\max }$ & $1.1 \mathrm{rad} \cdot \mu \mathrm{m}^{-1}$ \\
\hline$r_{e}$ & 60 & $\lambda_{\min }$ & $5.9 \mu \mathrm{m}^{-1}$ & $\lambda_{\max }$ & $15.4 \mu \mathrm{m}^{-1}$ \\
\hline$N_{f}$ & 3 & $z_{f}$ & {$[70,140,211] \mu \mathrm{m}$} & $\mathrm{NA}$ & 0.4 \\
\hline
\end{tabular}

Table 1: Parameters for point target simulations.

Similarly, $\overline{\mathbf{w}}^{\mathbf{q}}=\left(\Phi^{\mathbf{q}}\right)^{\mathrm{H}} \overline{\mathbf{y}}^{\mathbf{q}}$ consists of blocks $\hat{\mathbf{w}}_{s}^{\mathbf{q}}$ with $s \in N_{s}$, where the block is computed as

$$
\hat{\mathbf{w}}_{s}^{\mathbf{q}}=\sum_{f=1}^{N_{f}}\left(\hat{\mathbf{A}}_{f}^{\mathbf{q}}\right){ }^{\mathrm{H}} \mathbf{D}_{s}^{\mathrm{H}} \hat{\mathbf{y}}_{f}^{\mathbf{q}} .
$$

Many sparsity-promoting regularizers are non-differentiable. In this case, proximal methods such as FISTA [52 or the Alternating Direction Method of Multipliers (ADMM) [53 55] are attractive. This class of algorithms decomposes the problem [16 into a sequence of simpler subproblems. The solution of a linear system is often a key ingredient of such algorithms.

\section{Simulations}

We now describe two simulations used to validate the proposed approach. For simplicity, we consider only two spatial dimensions: one transverse $(x)$ and one axial $(z)$.

Preliminary work on the $N$-species model suffers from three unrealistic assumptions [15]. The simulations used unrealistic wavelength ranges, leading to nearly complete coverage of Fourier space. This removes the large null space present in $\mathbf{A}_{f}$ and simplifies the reconstruction problem. Secondly, the phantoms used satisfied the $N$ species model exactly; no spectral noise was considered. Finally, the synthetic data used in the simulations were generated using the asymptotic approximation to the ISAM operator, and thus under the first Born approximation. This neglects multiple scattering, absorption, and the discrepancy between the exact and approximate ISAM models. As a consequence, the simulations present an overly optimistic view of the proposed imaging modality.

We generate synthetic data using accurate physical models and system parameters. These data include multiple scattering and absorption effects—only the inversion is performed under the Born approximation. Further, the simulated targets do not precisely follow the $N$-species model; instead, there are position-dependent spectral variations within each species. In particular, we simulate an object of the form $\eta(\mathbf{r}, k)=\sum_{s=1}^{N_{s}} p_{s}(\mathbf{r}) h_{s}(\mathbf{r}, k)$, where $h_{s}(\mathbf{r}, k)=h_{s}(k)+e_{s}(\mathbf{r}, k)$ and $e_{s}(\mathbf{r}, k) \sim \mathcal{C N}\left(0, \xi_{s}\right)$ is a circular complex Gaussian random variable [56.

The minimization problem 16 is solved on an NVidia Titan X GPU using a combination of Python and CUDA 57, 58.

\subsection{Point Targets}

We formed a spectral library of five chemicals using refractive index data provided through the IARPA SILMARILS project. The corresponding spectral profiles are plotted in Fig. 8, The target consisted of 50 point scatterers. Each point scatterer is associated to one chemical species; only three species (out of the five possible) are present. We do not know a priori which chemicals are present.

We generated measurements using the Foldy-Lax model, which includes multiple scattering effects [59]. Data were generated at three focal planes in a $420 \times 280 \mu \mathrm{m}$ volume according to the parameters in Table 1 . The source power spectrum was flat over $\left[k_{\min }, k_{\max }\right]$. This combination of parameters-three active species, three focal planes, and a library of five possible species_corresponds to the case of (P2)

To assess the deviation from the single scattering model, we generated two sets of measurements using the same target. The first set of measurements, denoted s, uses the Foldy-Lax method and incorporates multiple scattering. The second, $\mathbf{s}_{B}$, is generated using the Born approximation and thus includes only single scattering events. The ratio $\left\|\mathbf{s}-\mathbf{s}_{B}\right\|_{2} /\left\|\mathbf{s}_{B}\right\|_{2}$ indicates that more than $20 \%$ of the energy in s comes from multiple scattering events.

We performed two sets of simulations: the first using Tikhonov regularization and the second using sparsitypromoting regularization. In the latter case, motivated by the spatial-domain sparsity of the target, we set $R(\mathbf{P})=$ 


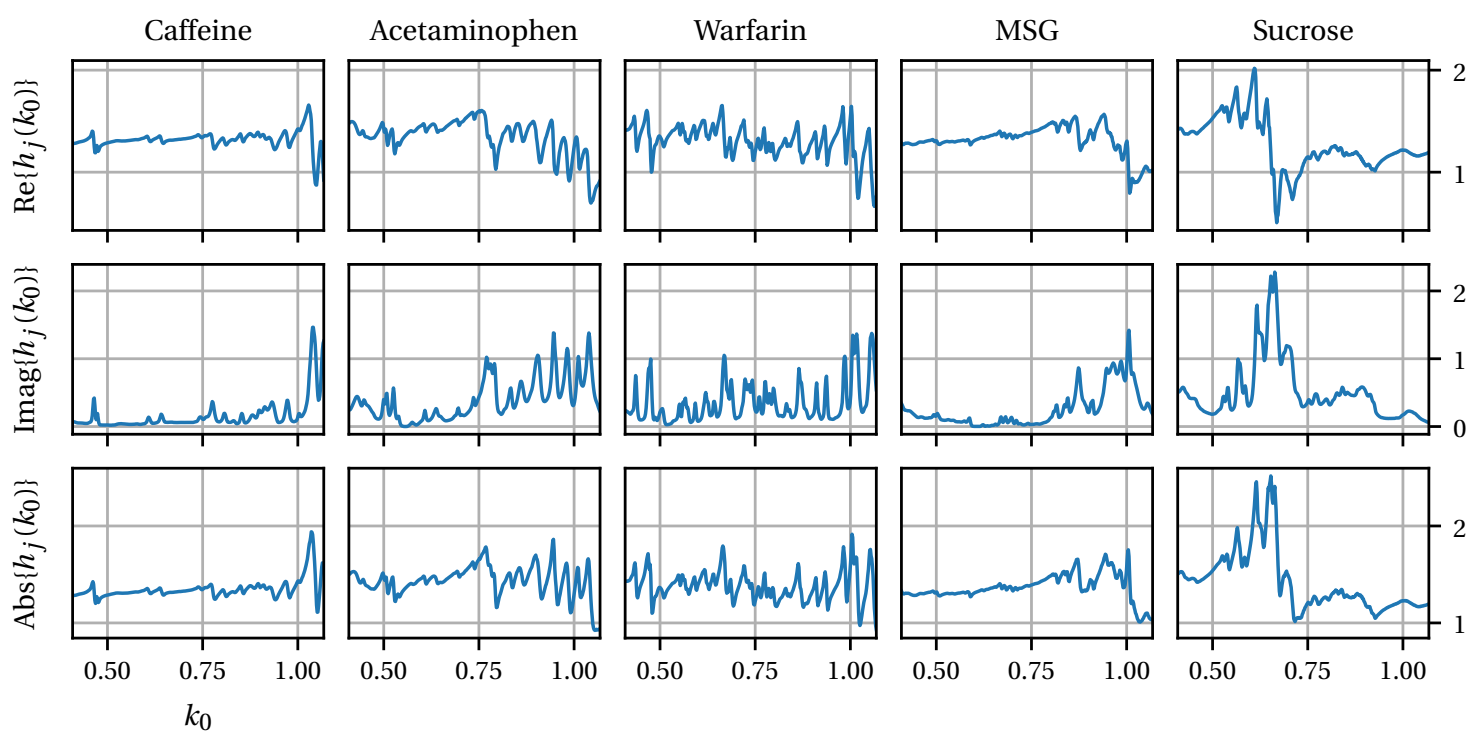

Figure 8: Spectral profiles for the five chemicals used in point scattering simulations.

$\sum_{s=1}^{5}\left\|\mathbf{p}_{s}\right\|_{1}$. In the Tikhonov case, we performed 300 iterations of conjugate gradient on the normal equations with $\lambda_{r}=10^{-5}$. In the case of $\ell_{1}$ regularization, we used 2000 iterations of the FISTA algorithm with $\lambda_{r}=10^{-3}$. Both cases terminated in under one minute.

The magnitude of the reconstructed spatial densities are shown in Fig.9 9 Recall that the surface of observable Fourier components is restricted to $k_{z}<0$. As such, any linear reconstruction method (e.g., Tikhonov-regularized least squares) will produce a complex-valued image; we display only the magnitude and squared magnitude of the recovered signal. For visualization purposes we have projected the point-target phantom onto the optical passband. In both cases, the reconstructed targets are correctly spatially localized and identified with the correct species.

The Tikhonov regularized reconstruction consists of the point scatterers sitting on top of a "noisy" background. The background is primarily due to multiple scattering effects and spectral variations which are not captured by the forward model. This background term is distributed across all five possible species; however, the recovered point scatterers are associated to the correct species. The background is eliminated when viewing the squared modulus of the reconstruction.

The $\ell_{1}$ regularized reconstruction suppresses the background term. There is nearly perfect agreement between the true target and the reconstructed target, despite taking data at only three, rather than five, focal planes. The sparsity of the target, coupled with the $\ell_{1}$ regularization, successfully eliminates artifacts due to multiple scattering.

For visualization purposes we map the three active species to the red, green, and blue channels of an RGB image. The filtered phantom, Tikhonov, and filtered $\ell_{1}$ reconstructions are shown in Fig. 10 .

\subsection{Cell Phantom}

\begin{tabular}{|cc||cc||cc|}
\hline$N_{x}$ & 256 & $L_{x}$ & $150.0 \mu \mathrm{m}$ & $\Delta_{x}$ & $0.6 \mu \mathrm{m}$ \\
\hline$N_{z}$ & 256 & $L_{z}$ & $150.0 \mu \mathrm{m}$ & $\Delta_{z}$ & $0.6 \mu \mathrm{m}$ \\
\hline$N_{k}$ & 256 & $k_{\min }$ & $0.7 \mathrm{rad} \cdot \mu \mathrm{m}^{-1}$ & $k_{\max }$ & $2.1 \mathrm{rad} \cdot \mu \mathrm{m}^{-1}$ \\
\hline$r_{e}$ & 67 & $\lambda_{\min }$ & $3.0 \mu \mathrm{m}^{-1}$ & $\lambda_{\max }$ & $9.0 \mu \mathrm{m}^{-1}$ \\
\hline$N_{f}$ & 3 & $z_{f}$ & {$[54,75,96] \mu \mathrm{m}$} & NA & 0.5 \\
\hline
\end{tabular}

Table 2: Parameters for cell phantom simulation. 


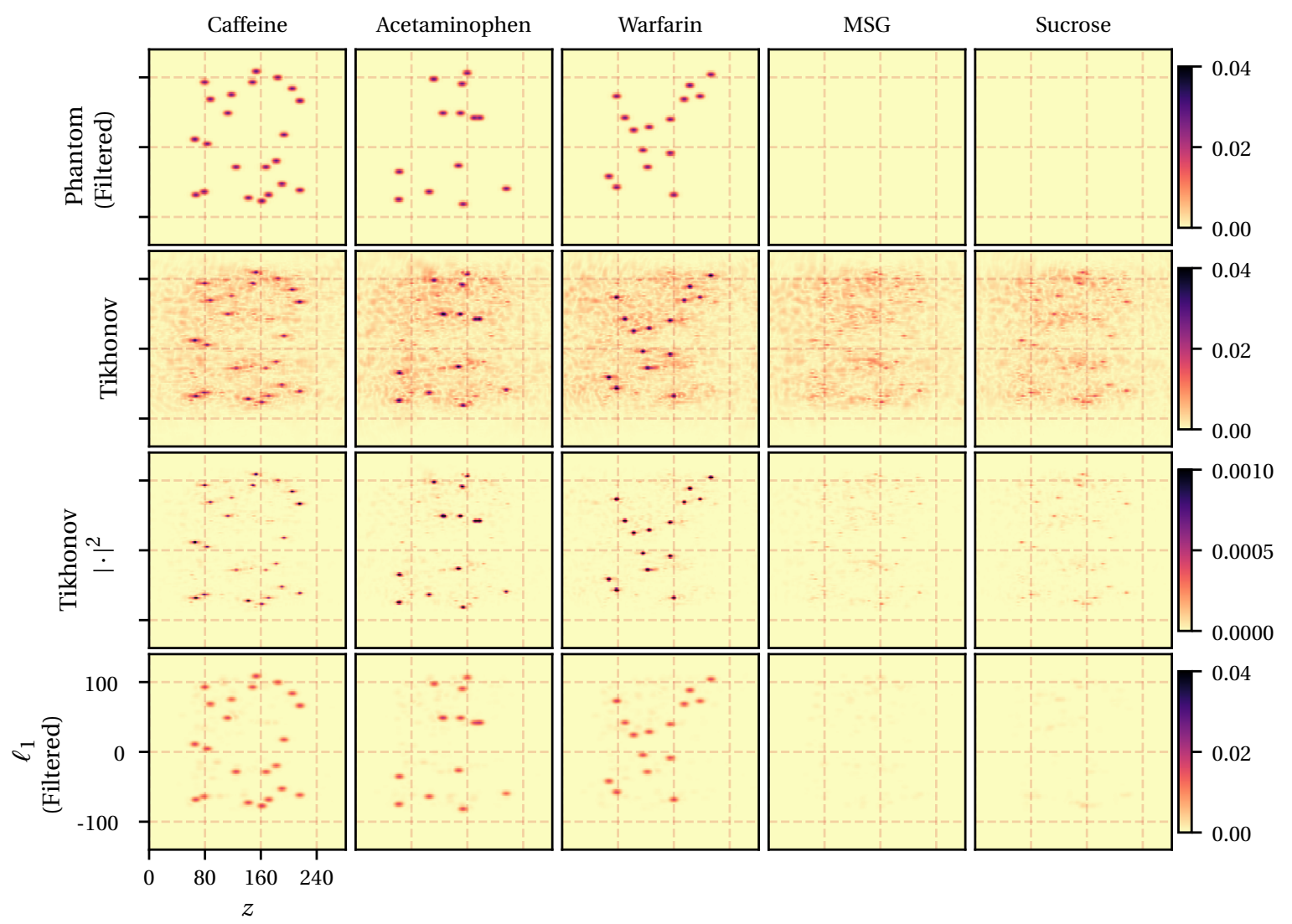

Figure 9: Reconstructions of point scatterers described in Section 6.1

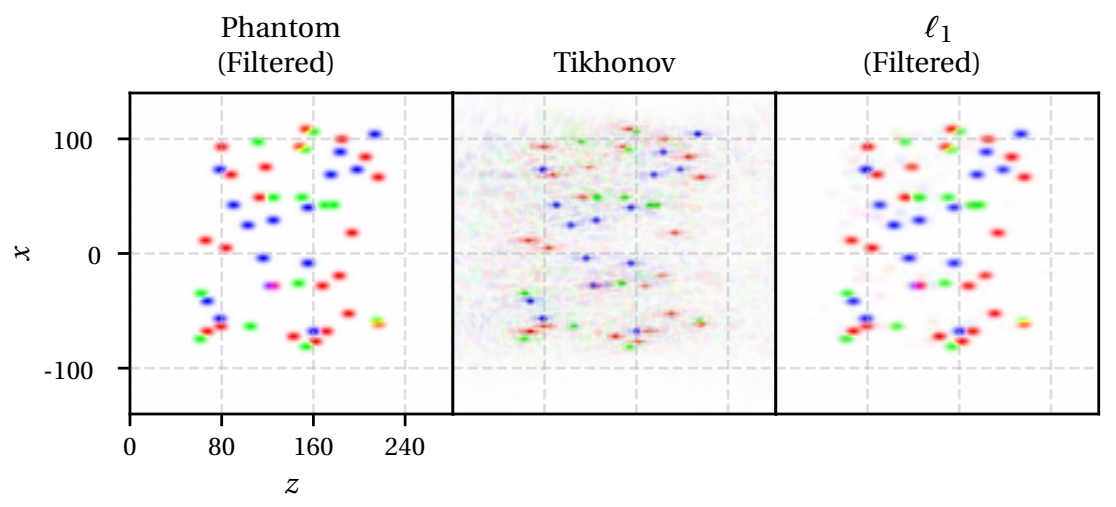

Figure 10: Visualizing the reconstructed point targets by assigning the three active species to a RGB channel. Red: caffeine, Green: acetaminophen, Blue: warfarin. The two species that are not present (MSG, Sucrose) were ignored. 
Next, we evaluated the ability to image extended targets. The target is the cellular phantom shown in Fig.11a which comprises three chemical species. The spectral library contains five total species.

We generated synthetic measurements by solving the Lipmann-Schwinger equation (see, e.g., [59]) using the using the Multi-Level Fast Multipole Algorithm (MLFMA) |60|. The data are not generated under the Born approximation, and thus includes multiple scattering and absorption phenomenon not captured using the forward model. We use a version of the MLFMA specialized for simulating two spatial dimensions [61 62].

We generated measurements for only three focal planes; the relevant computational parameters are listed in Table 2. We generated synthetic spectral profiles using a sum-of-Lorentzians model [63]. Each spectral profile is of the form

$$
h(k) \triangleq \sigma_{0}+\sum_{n=1}^{99} \frac{\sigma_{n}}{v_{n}^{2}-k^{2}-\mathrm{i} \gamma_{n} k},
$$

with $\sigma \sim \operatorname{Unif}[0,0.1], v \sim \operatorname{Unif}[1.2 \pi, 4.4 \pi]$, and $\gamma \sim \operatorname{Unif}\left[2 \pi \times 10^{-3}, 4 \pi \times 10^{-2}\right]$, where Unif $[a, b]$ is the uniform distribution over the interval $[a, b]$. The spectral profiles are plotted in Fig. $11 \mathrm{~b}$

The first-order Born approximation is valid only if the total phase change between the incident field and the field inside the sample is less than $\pi$-this implies that the object should be either weakly scattering or small in spatial extent 64 65. The proposed phantom is neither. To investigate the effect on scattering strength on the reconstructed images, we generated synthetic measurements for the scaled object $\delta \eta\left(\mathbf{r}_{0}, k\right)$ where $0<\delta \leq$ 1 . By reducing $\delta$, we reduce the scattering strength and eventually fall into a regime where the first-order Born approximation holds.

We used Tikhonov regularization with $\lambda_{r}=1 \times 10^{-4}$ and 500 iterations of the conjugate-gradient algorithm. The resulting reconstructions are shown in Fig.12. The top row illustrates the projection of the phantom onto the optical passband; this serves as the "gold standard" for Tikhonov-regularized reconstructions. The remaining rows are the reconstructed images. As expected, only the edges of the phantom that are nearly perpendicular to the optical axis are visible. The reconstructed images deteriorate as $\delta$ increases, particularly at the rear edge of each feature. However, the correct species is identified in each case; negligible energy is deposited into Species 4 and 5.

Fig. 13 illustrates the influence of the regularization parameter $\lambda_{r}$. Noise dominates the reconstruction when $\lambda_{r}$ is too small. When $\lambda_{r}$ is too large, there is no chemical identification- the recovered spatial densities are nearly identical for each species.

\section{Conclusions}

We have considered the problem of chemically specific and spatially resolved tomographic imaging from interferometric measurements. We require the target to be the linear combination of a finite number of distinct chemical species given data at a small number of $e$-face focal planes. We developed necessary and sufficient conditions for unique recovery of a target satisfying this model. Linear independence of the chemical spectra is not sufficientadditional spectral diversity is required.

In this paper, we assume the chemical spectra were either known or drawn from a library of possible spectra. In the latter case, the number of required focal planes scales with the number of chemicals present in the sample, not the total number in the library. Future work will consider extension fully blind problem.

Our approach requires interferometric (phase-resolved) measurements and solves the linearized scattering problem. This extension to intensity-only measurements and the removal of the Born approximation are two avenues for future work.

Phaseless, intensity-only diffraction tomography has been demonstrated by modifying the acquisition scheme [66-68] and by optimization-based approaches [69]. Advances in high performance computing [61 62 70] and deep learning $[71-73]$ have facilitated the solution of large scale inverse scattering problems without linearization. In some cases, solving the nonlinear inverse scattering problem overcomes the "missing cone" effect that hampers reconstruction of extended targets. However, thus far, these approaches have only considered non-dispersive objects. Extension of these methods to spectroscopic tomography within the $N$-species approximation is an exciting area of future work. 


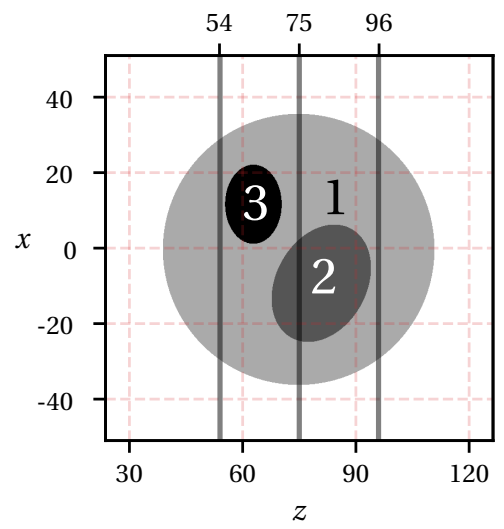

(a)
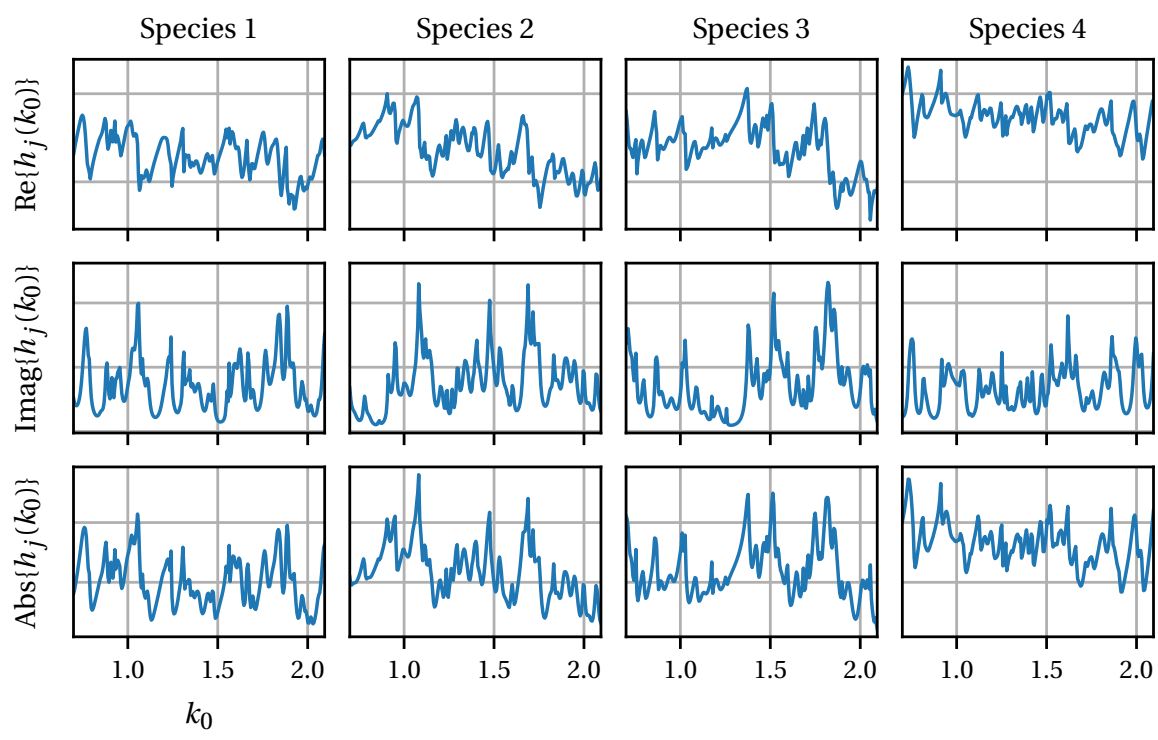

Species 5
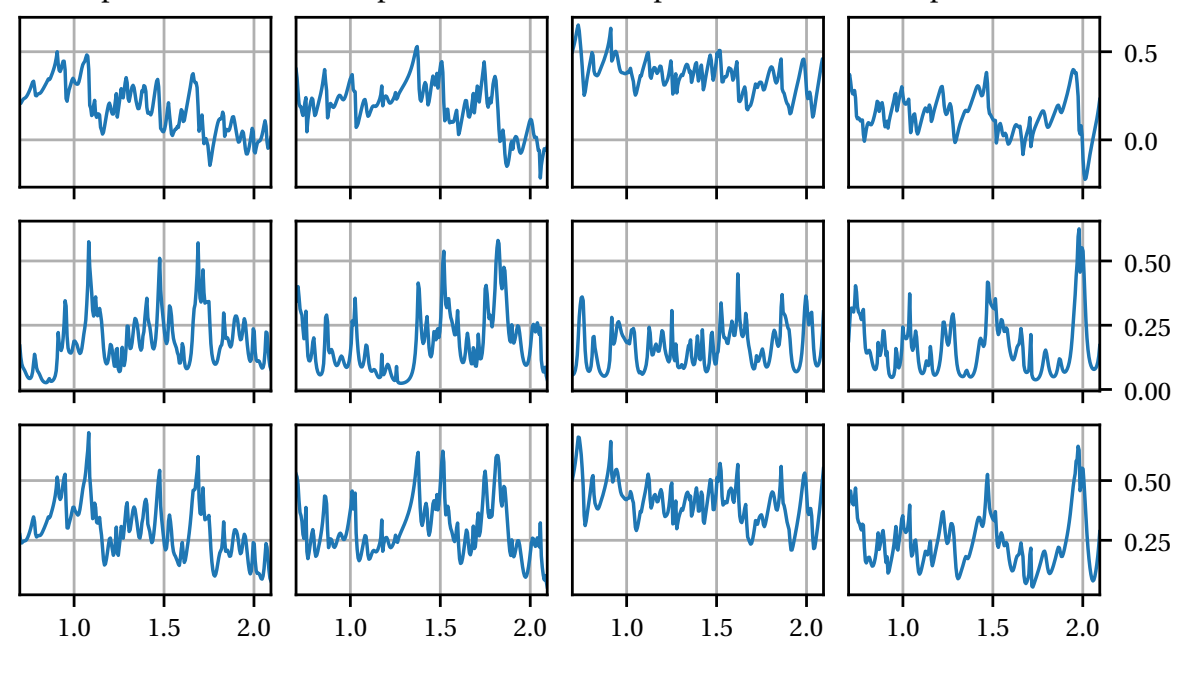

(b)

Figure 11: (a) Three-species cell phantom. The detector plane is plane located at $z=0$. Solid vertical lines denote the three focal planes. All units are $\mu \mathrm{m}$. (b)Spectral profiles for cell phantom, plotted for $\delta=1$. 


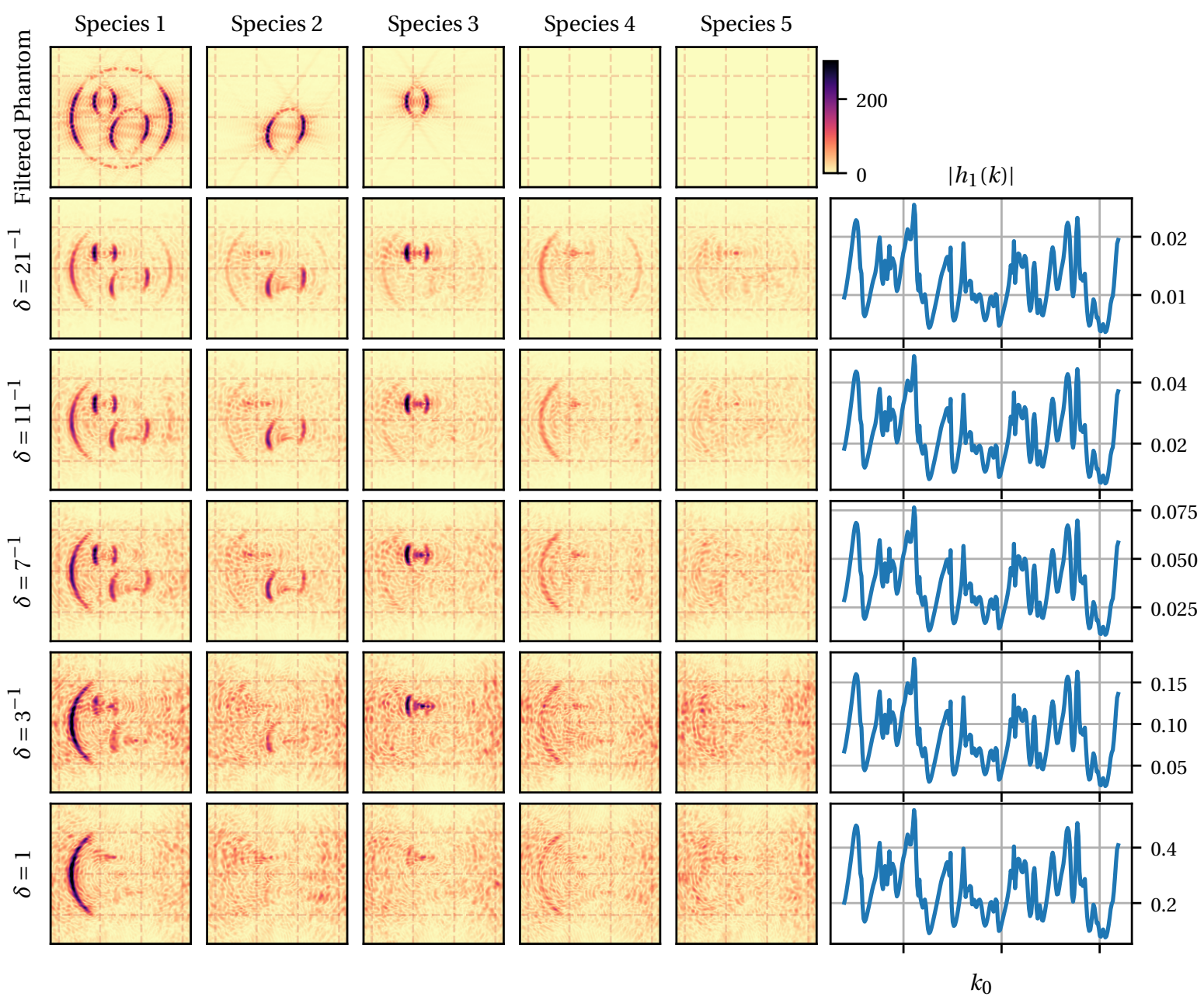

Figure 12: Reconstructions of cell phantom as a function of scattering strength. All reconstructions use Tikhonov regularization with $\lambda_{r}=10^{-5}$. 


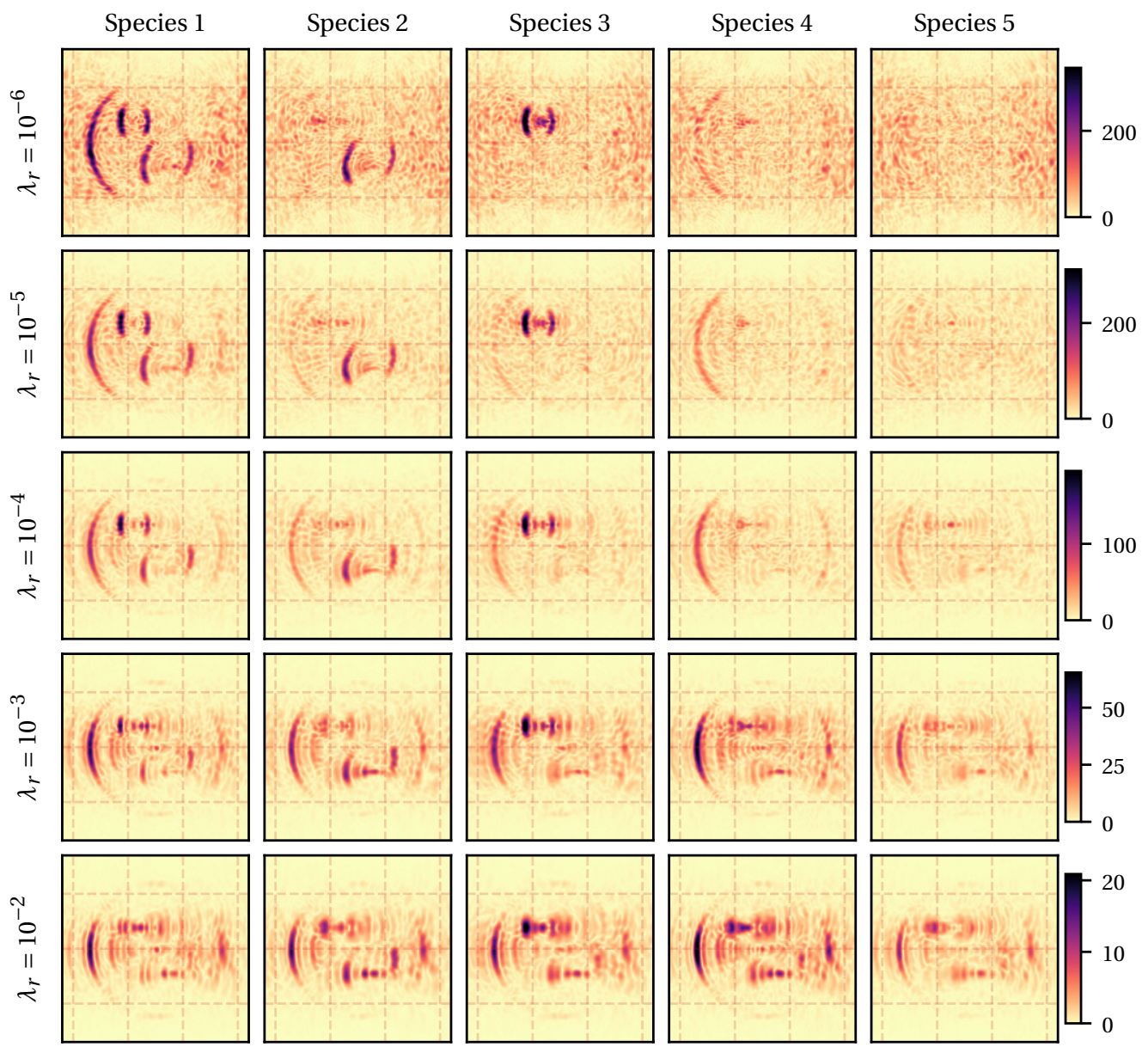

Figure 13: Reconstructions of cellular phantom using Tikhonov regularization and various levels of $\lambda_{r}$. The scattering strength parameter is $\delta=11^{-1}$. As $\lambda_{r}$ increases, the reconstruction fails to distinguish between chemical species. 


\section{Acknowledgments}

The work of L. Pfister was partially supported by the Andrew T. Yang fellowship. This research is based upon work partially supported by the Office of the Director of National Intelligence (ODNI), Intelligence Advanced Research Projects Activity (IARPA), via Air Force Research Laboratory (AFRL) FA8650-16-C- 9108. The views and conclusions contained herein are those of the authors and should not be interpreted as necessarily representing the official policies or endorsements, either expressed or implied, of the ODNI, IARPA, AFRL or the U.S. Government. The U.S. Government is authorized to reproduce and distribute reprints for Governmental purposes notwithstanding any copyright annotation thereon.

\section{A Proof of Main Theorems}

Proof of Lemma $1,(\mathrm{C} 1) \Longrightarrow(\mathrm{C} 2)$ Let $\overline{\mathbf{p}}^{\mathbf{q}} \in(\overline{\mathrm{N}} \mathbf{q})^{\perp}$ be the unique solution to $\overline{\mathbf{s}}^{\mathbf{q}}=\Phi^{\mathbf{q}} \overline{\mathbf{p}}^{\mathbf{q}}$. Let $\mathbf{x} \in \operatorname{null}\left\{\Phi^{\mathbf{q}}\right\} \cap\left(\overline{\mathrm{N}}^{\mathbf{q}}\right)^{\perp}$. Now $\Phi^{\mathbf{q}}\left(\overline{\mathbf{p}}^{\mathbf{q}}+\mathbf{x}\right)=\Phi^{\mathbf{q}} \overline{\mathbf{p}}^{\mathbf{q}}=\overline{\mathbf{s}}^{\mathbf{q}}$. As $\mathbf{x}+\overline{\mathbf{p}}^{\mathbf{q}} \in(\overline{\mathrm{N}} \mathbf{q})^{\perp}$, by $\left.(\mathrm{C} 1)\right] \mathbf{x}=0$. Thus $(\mathrm{C} 1) \Longrightarrow(\mathrm{C} 2)$

(C2) $\Longrightarrow(\mathrm{C} 3)$ Recall $\tilde{\Phi}^{\mathbf{q}}=\Phi^{\mathbf{q}}\left(\mathbf{I}_{N_{f}} \otimes \mathbf{V}^{\mathbf{q}}\right) \in \mathbb{C}^{N_{f} N_{k} \times N_{s} r}$. As $\mathbf{I}_{N_{f}} \otimes \mathbf{V}^{\mathbf{q}}$ is a basis for $(\overline{\mathrm{N}} \mathbf{q})^{\perp}$, and null $\left\{\Phi^{\mathbf{q}}\right\}=\overline{\mathrm{N}} \mathbf{q}$ by assumption, $\Phi^{\mathbf{q}} \mathbf{X}=\mathbf{0}$ if and only if $\mathbf{x}=\mathbf{0}$; thus null $\left\{\tilde{\Phi}^{\mathbf{q}}\right\}=\{\mathbf{0}\}$. By the rank nullity theorem, $\operatorname{rank}\left(\tilde{\Phi}^{\mathbf{q}}\right)=N_{s} r$.

(C3) $\Longrightarrow$ (C1) Suppose $\exists \mathbf{u}, \mathbf{v} \in(\overline{\mathrm{N}} \mathbf{q})^{\perp}$ such that $\Phi^{\mathbf{q}} \mathbf{u}=\Phi^{\mathbf{q}} \mathbf{v}$. As $\mathbf{I}_{N_{f}} \otimes \mathbf{V}^{\mathbf{q}}$ is a basis for $(\overline{\mathrm{N}} \mathbf{q})^{\perp}$, there are unique vectors $\mathbf{x}, \mathbf{y}$ such that $\mathbf{u}=\left(\mathbf{I}_{N_{f}} \otimes \mathbf{V}^{\mathbf{q}}\right) \mathbf{x}$ and $\mathbf{v}=\left(\mathbf{I}_{N_{f}} \otimes \mathbf{V}^{\mathbf{q}}\right) \mathbf{y}$. Now $\mathbf{0}=\Phi^{\mathbf{q}}(\mathbf{u}-\mathbf{v})=\tilde{\Phi}^{\mathbf{q}}(\mathbf{x}-\mathbf{y}) \Longrightarrow \mathbf{x}=\mathbf{y}$ as $\tilde{\Phi}^{\mathbf{q}}$ is full column rank; thus $\mathbf{u}=\mathbf{v}$, completing the proof.

The following lemma regarding the rank of the Khatri-Rao product will prove useful:

Lemma 3. Given $\mathbf{A} \in \mathbb{C}^{m \times n_{1}}$ and $\mathbf{B} \in \mathbb{C}^{m \times n_{2}}, \operatorname{rank}(\mathbf{A} \odot \mathbf{B}) \leq \min (m, \operatorname{rank}(\mathbf{A}) \operatorname{rank}(\mathbf{B}))$.

Proof. As $\mathbf{A} \odot \mathbf{B} \in \mathbb{C}^{m \times n_{1} n_{2}}$, we have $\operatorname{rank}(\mathbf{A} \odot \mathbf{B}) \leq \min \left(m, n_{1} n_{2}\right)$. Note that $\mathbf{A} \odot \mathbf{B}$ contains a subset of rows of the matrix $\mathbf{A} \otimes \mathbf{B}$. As the rank of the Kronkecker product is equal to the product of the ranks of $\mathbf{A}$ and $\mathbf{B}($ e.g., $[74])$, we have $\operatorname{rank}(\mathbf{A} \odot \mathbf{B}) \leq \operatorname{rank}(\mathbf{A} \otimes \mathbf{B})=\operatorname{rank}(\mathbf{A}) \operatorname{rank}(\mathbf{B})$.

Proof of Theorem [1. Here, we suppress the superscript q. By Lemma1 it suffices to show that the proposed conditions are necessary for $\tilde{\Phi}$ to have rank $N_{s} r$. (N1) follows as $\tilde{\Phi}$ can have rank $N_{s} r$ only if $N_{k} N_{f} \geq N_{s} r$.

We show (N2) by contradiction; suppose $\operatorname{rank}(\mathbf{H})=q<N_{s}$. By construction $\operatorname{rank}(\overline{\mathbf{H}})=\operatorname{rank}(\mathbf{H})$. Thus by Lemma $3 \operatorname{rank}(\Phi) \leq \operatorname{rank}(\overline{\mathbf{H}}) \operatorname{rank}\left(\overline{\mathbf{A}}^{\mathbf{q}}\right) \leq r q<N_{s} r$.

For (N3), suppose the first row of $\hat{\mathbf{B}}$ is orthogonal to the remaining $N_{k} N_{f}$ rows. Let $\mathbf{x}$ be a column vector formed from first row of $\hat{\mathbf{B}}$ and let $\mathbf{e}_{1} \triangleq[1,0, \ldots, 0] \in \mathbb{C}^{N_{k} N_{f}}$; by construction, $\hat{\mathbf{B}} \mathbf{x}=\mathbf{e}_{1}$. Set $\alpha=\sum_{s=2}^{N_{s}} \mathbf{h}_{s}[1] / \mathbf{h}_{1}[1]$; then

$$
\tilde{\Phi}\left[-\alpha \mathbf{x}^{\top}, \mathbf{x}^{\top}, \ldots, \mathbf{x}^{\top}\right]^{\top}=\operatorname{diag}\left\{\sum_{s=2}^{N_{s}} \mathbf{h}_{s}-\alpha \mathbf{h}_{1}\right\} \mathbf{e}_{1}=\mathbf{0},
$$

and so $\operatorname{rank}(\Phi) \leq N_{s} r-1$.

To show (N4) suppose there is a subset $J$ with $|J| \geq N_{s}$ such that $\mathbf{H}[J,:] \in \mathbb{C}^{|J| \times N_{s}}$ is rank $N_{s}$ and the remaining rows, $\mathbf{H}\left[J^{c},:\right] \in \mathbb{C}^{N_{k}-|J| \times N_{s}}$ has rank $q<N_{s}$. Define $\tilde{\Phi}^{J} \in \mathbb{C}^{N_{f}|J| \times N_{s} r}$ to be the rows of $\tilde{\Phi}$ involving the rows of $\mathbf{H}$ indexed by $J$; that is,

$$
\tilde{\Phi}^{J}=\left[\begin{array}{c}
\mathbf{H}[J,:] \odot \hat{\mathbf{B}}_{1}[J,:] \\
\vdots \\
\mathbf{H}[J,:] \odot \hat{\mathbf{B}}_{N_{f}}[J,:]
\end{array}\right],
$$

and construct $\tilde{\Phi}^{J^{c}} \in \mathbb{C}^{N_{f}\left(N_{k}-|J|\right) \times N_{s} r}$ using the rows indexed by $J^{c}$. As both $\hat{\mathbf{B}}[J,:] \in \mathbb{C}^{N_{f}|J| \times r}$ and $\hat{\mathbf{B}}\left[J^{c},:\right] \in \mathbb{C}^{N_{f}\left(N_{k}-|J|\right) \times r}$ have rank at most $r$, by Lemma 3 we have

$$
\operatorname{rank}(\tilde{\Phi}) \leq \operatorname{rank}\left(\tilde{\Phi}^{J}\right)+\operatorname{rank}\left(\tilde{\Phi}^{J^{c}}\right) \leq \min \left(N_{f}|J|, N_{s} r\right)+\min \left(N_{f}\left(N_{k}-|J|\right), q r\right) \triangleq \beta .
$$

We wish to establish conditions such that $\beta \geq N_{s} r$. This is clearly true, regardless of $q$, when $N_{f}|J| \geq N_{s} r$. When $|J|<N_{s} r / N_{f}$, we have

$$
\beta=N_{f}|J|+\min \left(N_{f}\left(N_{k}-|J|\right), q r\right) .
$$


Suppose $N_{f}\left(N_{k}-|J|\right)<q r$; then $\beta=N_{f} N_{k} \geq N_{s} r$ where the inequality follows from condition (N1) Otherwise, if $N_{f}\left(N_{k}-|J|\right) \geq q r$, then $\beta=N_{f}|J|+q r$ and $q \geq N_{s}-N_{f}|J| / r$ implies $\beta \geq N_{s} r$.

To show (N5), for each $i \in\left[N_{k}\right]$ we define the index set $J_{i}=\left\{i, i+N_{k}, \ldots, i+\left(N_{f}-1\right) N_{k}\right\}$; now,

$$
\tilde{\Phi}^{J_{i}}=\left(\mathbb{1}_{N_{f}}^{\top} \otimes \overline{\mathbf{H}}\left[J_{i},:\right]\right) \odot \overline{\mathbf{B}}\left[J_{i},:\right]=\left[\begin{array}{cccc}
\mathbf{h}_{1}[i] \hat{\mathbf{B}}_{1}[i,:] & \mathbf{h}_{2}[i] \hat{\mathbf{B}}_{1}[i,:] & \ldots & \mathbf{h}_{N_{s}}[i] \hat{\mathbf{B}}_{1}[i,:] \\
\vdots & \vdots & & \vdots \\
\mathbf{h}_{1}[i] \hat{\mathbf{B}}_{N_{f}}[i,:] & \mathbf{h}_{2}[i] \hat{\mathbf{B}}_{N_{f}}[i,:] & \ldots & \mathbf{h}_{N_{s}}[i] \hat{\mathbf{B}}_{N_{f}}[i,:]
\end{array}\right] \in \mathbb{C}^{N_{f} \times N_{s} r}
$$

Now, $\operatorname{rank}(\tilde{\Phi}) \leq \sum_{i=1}^{N_{k}} \operatorname{rank}\left(\tilde{\Phi}^{J_{i}}\right) \leq \sum_{i=1}^{N_{k}} \operatorname{rank}\left(\hat{\mathbf{B}}\left[J_{i},:\right]\right)$, where the final inequality follows from Lemma $3 \operatorname{and} \operatorname{rank}\left(\left(\mathbb{1}_{N_{f}}^{\top} \otimes \mathbf{H}\left[J_{i},:\right]\right)\right)=$ 1. Setting this upper bound to $N_{s} r$ gives the statement.

Proof of Theorem 2. We omit the superscript q. It suffices to prove the case where $\tilde{\Phi}$ is square, $N_{k}=r$ and $N_{f}=N_{s}$. Then $\operatorname{rank}(\tilde{\Phi}) \in \mathbb{C}^{N_{s} r \times N_{s} r}=N_{s} r$ if and only if

$$
\theta(\mathbf{H}) \triangleq \operatorname{det} \tilde{\Phi}=\operatorname{det}[\overline{\mathbf{H}} \odot \overline{\mathbf{B}}] \neq 0 .
$$

Now, $\theta(\mathbf{H})$ is a multivariate polynomial in the entries of $\mathbf{H}$ whose coefficents depend only on the entries of $\overline{\mathbf{B}}$. Thus $\theta(\mathbf{H})$ is either identically zero or its zero set is an affine algebraic set and thus a nowhere dense set of measure zero. It suffices to show $\theta(\mathbf{H}) \neq 0$ for a single choice of $\mathbf{H}$ (see, e.g., [75- 77] and references therein).

We can permute the rows of $\tilde{\Phi}$ such that the first $N_{k}$ rows are indexed by $J_{1}$, the next $N_{k}$ rows by $J_{2}$, and so on. In particular, there is a permutation matrix $\Pi \in \mathbb{C}^{N_{k} N_{s} \times N_{k} N_{s}}$ such that (c.f. 14)

$$
\Pi \tilde{\Phi}=\left[\begin{array}{ccc}
\mathbf{D}_{1}\left[J_{1}, J_{1}\right] \hat{\mathbf{B}}_{1}\left[J_{1},:\right] & \ldots & \mathbf{D}_{N_{f}}\left[J_{1}, J_{1}\right] \hat{\mathbf{B}}_{1}\left[J_{1},:\right] \\
\vdots & & \vdots \\
\mathbf{D}_{1}\left[J_{1}, J_{1}\right] \hat{\mathbf{B}}_{N_{f}}\left[J_{1},:\right] & \ldots & \mathbf{D}_{N_{f}}\left[J_{1}, J_{1}\right] \hat{\mathbf{B}}_{N_{f}}\left[J_{1},:\right] \\
\mathbf{D}_{1}\left[J_{2}, J_{2}\right] \hat{\mathbf{B}}_{1}\left[J_{2},:\right] & \ldots & \mathbf{D}_{N_{f}}\left[J_{2}, J_{2}\right] \hat{\mathbf{B}}_{1}\left[J_{2},:\right] \\
\vdots & \ddots & \vdots \\
\mathbf{D}_{1}\left[J_{N_{f}}, J_{N_{f}}\right] \hat{\mathbf{B}}_{N_{f}}\left[J_{N_{f}},:\right] & \ldots & \mathbf{D}_{N_{f}}\left[J_{N_{f}}, J_{N_{f}}\right] \hat{\mathbf{B}}_{N_{f}}\left[J_{N_{f}},:\right]
\end{array}\right]=\left[\begin{array}{ccc}
\check{\mathbf{D}}_{1}^{J_{1}} \mathbf{C}_{1} & \ldots & \check{\mathbf{D}}_{N_{f}}^{J_{1}} \mathbf{C}_{1} \\
\check{\mathbf{D}}_{1}^{J_{2}} \mathbf{C}_{2} & \ldots & \check{\mathbf{D}}_{N_{f}}^{J_{2}} \mathbf{C}_{2} \\
\vdots & \ddots & \vdots \\
\check{\mathbf{D}}_{1}^{J_{N_{f}}} \mathbf{C}_{N_{f}} & \ldots & \check{\mathbf{D}}_{N_{f}}^{J_{N_{f}}} \mathbf{C}_{N_{f}}
\end{array}\right],
$$

where, in an abuse of notation, we write $\check{\mathbf{D}}_{i}^{J}=\left(\mathbb{1}_{N_{f}}^{\top} \otimes \mathbf{D}_{i}[J, J]\right)$.

Next, we specify the choice of $\mathbf{H}$. By assumption, $r=N_{k}=m N_{f}$ for some integer $m$. For each $i \in\left[N_{s}\right]$, we set $\mathbf{h}_{i}\left[J_{i}\right]=\mathbf{1}_{m}$ and set remaining coordinates are set to zero. With this construction, $\check{\mathbf{D}}_{i}^{J_{j}}=\mathbf{I}_{m}$ if $i=j$; otherwise, $\check{\mathbf{D}}_{i}^{J_{j}}=\mathbf{0}_{m}$. Now

$$
\Pi \tilde{\Phi}=\left[\begin{array}{cccc}
\mathbf{C}_{1} & \mathbf{0}_{m} & \ldots & \mathbf{0}_{m} \\
\mathbf{0}_{m} & \mathbf{C}_{2} & \ldots & \mathbf{0}_{m} \\
\vdots & \vdots & \ddots & \vdots \\
\mathbf{0}_{m} & \mathbf{0}_{m} & \ldots & \mathbf{C}_{N_{f}}
\end{array}\right],
$$

that is, $\tilde{\Phi}$ is similar to a block diagonal matrix. As each block along the diagonal is full rank by assumption, $\tilde{\Phi}$ is full rank.

\section{References}

[1] R. Bhargava, "Infrared spectroscopic imaging: The next generation," Appl. Spectrosc., vol. 66, pp. 1091-1120, Oct. 2012.

[2] B. J. Davis, P. S. Carney, and R. Bhargava, "Theory of midinfrared absorption microspectroscopy: I. Homogeneous samples," Anal. Chem., vol. 82, pp. 3474-3486, May 2010.

[3] — - "Theory of midinfrared absorption microspectroscopy: II. Heterogeneous samples," Anal. Chem., vol. 82, pp. 3487-3499, May 2010. 
[4] R. K. Reddy, M. J. Walsh, M. V. Schulmerich, P. S. Carney, and R. Bhargava, "High-definition infrared spectroscopic imaging," Appl. Spectrosc., vol. 67, pp. 93-105, Jan 2013.

[5] S. Tiwari, J. Raman, V. Reddy, A. Ghetler, R. P. Tella, Y. Han, C. R. Moon, C. D. Hoke, and R. Bhargava, “Towards translation of discrete frequency infrared spectroscopic imaging for digital histopathology of clinical biopsy samples," Anal. Chem., vol. 88, pp. 10 183-10 190, 2016.

[6] S. Tiwari, "Development of stainless cardiac histology of clinical biopsy samples with infrared spectroscopy," Master's thesis, Univeristy of Illinois at Urbana Champaign, 2017.

[7] S. Mittal, K. Yeh, L. S. Leslie, S. Kenkel, A. Kajdacsy-Balla, and R. Bhargava, "Simultaneous cancer and tumor microenvironment subtyping using confocal infrared microscopy for all-digital molecular histopathology," Proceedings of the National Academy of Sciences, vol. 115, pp. E5651-E5660, 2018.

[8] A. F. Fercher, W. Drexler, C. K. Hitzenberger, and T. Lasser, "Optical coherence tomography-principles and applications," Reports on Progress in Physics, vol. 66, pp. 239-303, 2003.

[9] B. J. Davis, D. L. Marks, T. S. Ralston, P. S. Carney, and S. A. Boppart, "Interferometric synthetic aperture microscopy: Computed imaging for scanned coherent microscopy,” Sensors, vol. 8, pp. 3903-3931, Jun. 2008.

[10] T. S. Ralston, D. L. Marks, P. S. Carney, and S. A. Boppart, "Interferometric synthetic aperture microscopy," Nature Physics, vol. 3, pp. 129-134, 2007.

[11] B. J. Davis, S. C. Schlachter, D. L. Marks, T. S. Ralston, S. A. Boppart, and P. S. Carney, "Nonparaxial vectorfield modeling of optical coherence tomography and interferometric synthetic aperture microscopy," JOSA A, vol. 24, pp. 2527-2542, 2007.

[12] A. L. Oldenburg, C. Xu, and S. A. Boppart, "Spectroscopic optical coherence tomography and microscopy," IEEE J. Sel. Topics Quantum Electron., vol. 13, pp. 1629-1640, 2007.

[13] U. Morgner, W. Drexler, F. X. Kärtner, X. D. Li, C. Pitris, E. P. Ippen, and J. G. Fujimoto, "Spectroscopic optical coherence tomography," Opt. Lett., vol. 25, pp. 111-114, 2000.

[14] N. Bosschaart, T. G. van Leeuwen, M. C. G. Aalders, and D. J. Faber, "Quantitative comparison of analysis methods for spectroscopic optical coherence tomography," Biomedical Optics Express, vol. 4, pp. 2570-2584, 2013.

[15] B. Deutsch, R. Reddy, D. Mayerich, R. Bhargava, and P. S. Carney, "Compositional prior information in computational infrared spectroscopic imaging,” J. Opt. Soc. Am. A, vol. 32, pp. 1126-1131, Jun. 2015.

[16] T. S. Ralston, D. L. Marks, P. S. Carney, and S. A. Boppart, "Inverse scattering for optical coherence tomography," JOSA A, vol. 23, pp. 1027-1037, 2006.

[17] A. Sentenac and J. Mertz, "Unified description of three-dimensional optical diffraction microscopy: From transmission microscopy to optical coherence tomography: Tutorial," Journal of the Optical Society of America A, vol. 35, pp. 748-754, 2018.

[18] R. Wu and M. N. Toksöz, "Diffraction tomography and multisource holography applied to seismic imaging," GEOPHYSICS, vol. 52, pp. 11-25, 1987.

[19] A. C. Kak and M. Slaney, Principles of Computerized Tomographic Imaging. Society of Industrial and Applied Mathematics, 2001.

[20] Z. Pei Liang, "Spatiotemporal imaging with partially separable functions," in 2007 IEEE International Symposium on Biomedical Imaging: From Nano to Macro, - 2007, pp. 988-991.

[21] H. Nguyen, J. Haldar, M. Do, and Z.-P. Liang, "Denoising of MR spectroscopic imaging data with spatialspectral regularization," in 2010 IEEE International Symposium on Biomedical Imaging: From Nano to Macro, 2010, pp. 720-723. 
[22] H. Nguyen, X. Peng, M. Do, and Z.-P. Liang, "Spatiotemporal denoising of MR spectroscopic imaging data by low-rank approximations," in IEEE International Symposium on Biomedical Imaging: From Nano to Macro, 2011, pp. 857-860.

[23] — - "Denoising MR spectroscopic imaging data with low-rank approximations," IEEE Trans. Biomed. Eng., vol. 60, pp. 78-89, 2013.

[24] R. E. Alvarez and A. Macovski, "Energy-selective reconstructions in X-ray computerised tomography," Physics in Medicine and Biology, vol. 21, pp. 733-744, 1976.

[25] J. Cammin, J. S. Iwanczyk, and K. Taguchi, "Spectral/photon-counting computed tomography," in Emerging Imaging Technologies in Medicine. CRC Press, 2012, pp. 40-57.

[26] R. Kress, Linear Integral Equations, ser. Applied Mathematical Sciences. Springer New York, 2014.

[27] H. Barrett, Foundations of Image Science. Hoboken, NJ: Wiley-Interscience, 2004.

[28] H. J. Landau and H. O. Pollak, "Prolate spheroidal wave functions, Fourier analysis and uncertainty-III: The dimension of the space of essentially time- and band-limited signals," Bell System Technical Journal, vol. 41, pp. 1295-1336, 1962.

[29] D. Slepian and H. O. Pollak, "Prolate spheroidal wave functions, Fourier analysis and uncertainty - I," Bell System Technical Journal, vol. 40, pp. 43-63, 1961.

[30] H. J. Landau and H. O. Pollak, "Prolate spheroidal wave functions, Fourier analysis and uncertainty - II," Bell System Technical Journal, vol. 40, pp. 65-84, 1961.

[31] D. Slepian, "Prolate spheroidal wave functions, Fourier analysis, and uncertainty-V: The discrete case," Bell System Technical Journal, vol. 57, pp. 1371-1430, 1978.

[32] _ - "Some comments on Fourier analysis, uncertainty and modeling," SIAM Review, vol. 25, pp. 379-393, 1983.

[33] A. Jain and S. Ranganath, "Extrapolation algorithms for discrete signals with application in spectral estimation," IEEE Transactions on Acoustics, Speech, and Signal Processing, vol. 29, pp. 830-845, 1981.

[34] F. A. Grünbaum, "Eigenvectors of a Toeplitz matrix: Discrete version of the prolate spheroidal wave functions," SIAM Journal on Algebraic Discrete Methods, vol. 2, pp. 136-141, 1981.

[35] Z. Zhu, S. Karnik, M. A. Davenport, J. Romberg, and M. B. Wakin, "The eigenvalue distribution of discrete periodic time-frequency limiting operators,” IEEE Signal Processing Letters, vol. 25, pp. 95-99, 2018.

[36] Y. Xu, X. K. B. Chng, S. G. Adie, S. A. Boppart, and P. S. Carney, "Multifocal interferometric synthetic aperture microscopy," Opt. Express, vol. 22, pp. 16 606-16 618, 2014.

[37] E. Wolf and M. Nieto-Vesperinas, "Analyticity of the angular spectrum amplitude of scattered fields and some of its consequences," Journal of the Optical Society of America A, vol. 2, pp. 886-890, 1985.

[38] A. J. Devaney, "Nonuniqueness in the inverse scattering problem," Journal of Mathematical Physics, vol. 19, pp. 1526-1531, 1978.

[39] N. D. Sidiropoulos and R. Bro, "On the uniqueness of multilinear decomposition of n-way arrays," Journal of Chemometrics, vol. 14, pp. 229-239, 2000.

[40] S. Khanna and C. R. Murthy, "Corrections to 'On the restricted isometry of the columnwise Khatri-Rao product'," IEEE Transactions on Signal Processing, vol. 67, pp. 2387-2388, 2019.

[41] — - "On the restricted isometry of the columnwise Khatri-Rao product," IEEE Transactions on Signal Processing, vol. 66, pp. 1170-1183, 2018.

[42] A. Fengler and P. Jung, "On the restricted isometry property of centered self Khatri-Rao products," CoRR, 2019, arXiv:1905.09245 [cs.IT] 
[43] E. S. Allman, C. Matias, and J. A. Rhodes, "Identifiability of parameters in latent structure models with many observed variables," The Annals of Statistics, vol. 37, pp. 3099-3132, 2009.

[44] A. Bhaskara, M. Charikar, A. Moitra, and A. Vijayaraghavan, "Smoothed analysis of tensor decompositions," in Proceedings of the 46th Annual ACM Symposium on Theory of Computing - STOC '14, - 2014, pp. 594-603.

[45] A. E. Gamal, N. Naderializadeh, and A. S. Avestimehr, "When does an ensemble of matrices with randomly scaled rows lose rank?” in 2015 IEEE International Symposium on Information Theory (ISIT), 62015 , pp. 15021506.

[46] Y. C. Eldar and H. Bolcskei, "Block-sparsity: Coherence and efficient recovery," in 2009 IEEE International Conference on Acoustics, Speech and Signal Processing, Apr. 2009, pp. 2885-2888.

[47] Y. Eldar, P. Kuppinger, and H. Bolcskei, "Block-sparse signals: Uncertainty relations and efficient recovery," IEEE Trans. Signal Process., vol. 58, pp. 3042-3054, 2010.

[48] Y. Eldar and M. Mishali, "Robust recovery of signals from a structured union of subspaces," IEEE Trans. Inf. Theory, vol. 55, pp. 5302-5316, 2009.

[49] E. Elhamifar and R. Vidal, "Block-sparse recovery via convex optimization," IEEE Trans. Signal Process., vol. 60, pp. 4094-4107, 2012.

[50] T. S. Ralston, D. L. Marks, S. A. Boppart, and P. S. Carney, "Inverse scattering for high-resolution interferometric microscopy," Opt. Lett., vol. 31, pp. 3585-3587, 2006.

[51] H. Erdogan and J. Fessler, "Monotonic algorithms for transmission tomography," IEEE Trans. Med. Imag., vol. 18, pp. 801-14, Sep. 1999.

[52] A. Beck and M. Teboulle, "A fast iterative shrinkage-thresholding algorithm for linear inverse problems," SIAM Journal on Imaging Sciences, vol. 2, pp. 183-202, Jan. 2009.

[53] J. Eckstein and D. Bertsekas, "On the Douglas-Rachford splitting method and the proximal point algorithm for maximal monotone operators,” Mathematical Programming, vol. 55, pp. 293-318, 1992.

[54] S. Boyd, N. Parikh, E. Chu, B. Peleato, and J. Eckstein, "Distributed optimization and statistical learning via the alternating direction method of multipliers," Foundations and Trends in Machine Learning, vol. 3, pp. 1-122, 2010.

[55] M. V. Afonso, J. M. Bioucas-Dias, and M. A. T. Figueiredo, "An augmented Lagrangian approach to the constrained optimization formulation of imaging inverse problems," IEEE Trans. Image Process., vol. 20, pp. 681695, 2011.

[56] J. W. Goodman, Statistical Optics (Wiley Series in Pure and Applied Optics). Wiley, 2015.

[57] L. E. Givon, T. Unterthiner, N. B. Erichson, D. W. Chiang, E. Larson, L. Pfister, S. Dieleman, G. R. Lee, S. van der Walt, B. Menn, T. M. Moldovan, F. Bastien, X. Shi, J. Schlüter, B. Thomas, C. Capdevila, A. Rubinsteyn, M. M. Forbes, J. Frelinger, T. Klein, B. Merry, N. Merill, L. Pastewka, L. Y. Liu, S. Clarkson, M. Rader, S. Taylor, A. Bergeron, N. H. Ukani, F. Wang, W.-K. Lee, and Y. Zhou, "scikit-cuda 0.5.3: a Python interface to GPU-powered libraries," May 2019.

[58] A. Klöckner, N. Pinto, Y. Lee, B. Catanzaro, P. Ivanov, and A. Fasih, "PyCUDA and PyOpenCL: A scripting-based approach to GPU run-time code generation,” Parallel Computing, vol. 38, pp. 157-174, 2012.

[59] B. Anthony J. Devaney, Northeastern University, Mathematical Foundations of Imaging, Tomography and Wavefield Inversion. Cambridge University Press, 2012.

[60] R. Coifman, V. Rokhlin, and S. Wandzura, "The fast multipole method for the wave equation: A pedestrian prescription," IEEE Antennas and Propagation Magazine, vol. 35, pp. 7-12, 1993. 
[61] L. L. Meng, M. Hidayetoglu, T. Xia, W. E. I. Sha, L. J. Jiang, and W. C. Chew, "A wideband 2-d fast multipole algorithm with a novel diagonalization form," IEEE Transactions on Antennas and Propagation, vol. 66, pp. 7477-7482, 2018.

[62] M. Hidayetoglu, C. Pearson, I. E. Hajj, L. Gurel, W. C. Chew, and W.-M. Hwu, "A fast and massively-parallel inverse solver for multiple-scattering tomographic image reconstruction," in 2018 IEEE International Parallel and Distributed Processing Symposium (IPDPS), 5 2018, pp. 64-74.

[63] G. Fowles, Introduction to Modern Optics. New York: Dover Publications, 1989.

[64] B. Chen and J. J. Stamnes, "Validity of diffraction tomography based on the first Born and the first Rytov approximations," Appl. Opt., vol. 37, pp. 2996-3006, 1998.

[65] M. Slaney, A. Kak, and L. Larsen, "Limitations of imaging with first-order diffraction tomography," IEEE Trans. Microw. Theory Techn., vol. 32, pp. 860-874, 1984.

[66] M. A. Anastasio, D. Shi, and G. Gbur, "Multispectral intensity diffraction tomography reconstruction theory: Quasi-nondispersive objects," Journal of the Optical Society of America A, vol. 23, pp. 1359-1368, 2006.

[67] M. A. Anastasio, Q. Xu, and D. Shi, "Multispectral intensity diffraction tomography: Single material objects with variable densities," Journal of the Optical Society of America A, vol. 26, pp. 403-412, 2009.

[68] R. Horstmeyer, J. Chung, X. Ou, G. Zheng, and C. Yang, "Diffraction tomography with Fourier ptychography," Optica, vol. 3, pp. 827-835, 2016.

[69] T.-A. Pham, E. Soubies, A. Goy, J. Lim, F. Soulez, D. Psaltis, and M. Unser, "Versatile reconstruction framework for diffraction tomography with intensity measurements and multiple scattering," Optics Express, vol. 26, pp. 2749-2763, 2018.

[70] M. Hidayetoglu, W.-M. Hwu, and W. C. Chew, "Supercomputing for full-wave tomographic image reconstruction in near-real time," in 2018 IEEE International Symposium on Antennas and Propagation \& USNC/URSI National Radio Science Meeting, 7 2018, pp. 1841-1842.

[71] H.-Y. Liu, D. Liu, H. Mansour, P. T. Boufounos, L. Waller, and U. S. Kamilov, "SEAGLE: Sparsity-driven image reconstruction under multiple scattering," IEEE Transactions on Computational Imaging, vol. 4, pp. 73-86, 2018.

[72] Y. Sun, Z. Xia, and U. S. Kamilov, "Efficient and accurate inversion of multiple scattering with deep learning," Optics Express, vol. 26, pp. 14678-14688, 2018.

[73] E. Soubies, T.-A. Pham, and M. Unser, "Efficient inversion of multiple-scattering model for optical diffraction tomography," Optics Express, vol. 25, pp. 21 786-21 800, 2017.

[74] G. H. Golub and C. F. V. Loan, Matrix Computations. Johns Hopkins University Press, 2012.

[75] G. Harikumar and Y. Bresler, "FIR perfect signal reconstruction from multiple convolutions: Minimum deconvolver orders," IEEE Transactions on Signal Processing, vol. 46, pp. 215-218, 1998.

[76] — - "Perfect blind restoration of images blurred by multiple filters: Theory and efficient algorithms," IEEE Transactions on Image Processing, vol. 8, pp. 202-219, 1999.

[77] T. Jiang, N. Sidiropoulos, and J. ten Berge, "Almost-sure identifiability of multidimensional harmonic retrieval," IEEE Transactions on Signal Processing, vol. 49, pp. 1849-1859, 2001. 